\title{
Squirrel-Cage Fan System Optimization and Flow Field Prediction Using Parallel Filling Criterion and Surrogate Model
}

\author{
Qianhao Xiao ${ }^{1}$, Xuna Shi ${ }^{2}$, Linghui $\mathrm{Wu}^{2}{ }^{2}$, Jun Wang ${ }^{1, *}$, Yanyan Ding ${ }^{1}$ and Boyan Jiang ${ }^{1}$ \\ 1 School of Energy and Power Engineering, Huazhong University of Science and Technology, Luoyu Road \\ No.1037, Wuhan 430074, China; d202080465@hust.edu.cn (Q.X.); d201880387@hust.edu.cn (Y.D.); \\ jiangby@hust.edu.cn (B.J.) \\ 2 Zhejiang Key Laboratory of Health Smart Kitchen System Integration, Ningbo Fangtai Kitchenware Co., Ltd., \\ 218 Binhai 2nd Road, Ningbo 315336, China; shixn@fotile.com (X.S.); wulhd@fotile.com (L.W.) \\ * Correspondence: wangjhust@hust.edu.cn
}

check for updates

Citation: Xiao, Q.; Shi, X.; Wu, L.; Wang, J.; Ding, Y.; Jiang, B. Squirrel-Cage Fan System Optimization and Flow Field Prediction Using Parallel Filling Criterion and Surrogate Model. Processes 2021, 9, 1620. https:// doi.org/10.3390/pr9091620

Academic Editor:

Farhad Ein-Mozaffari

Received: 26 July 2021

Accepted: 7 September 2021

Published: 8 September 2021

Publisher's Note: MDPI stays neutral with regard to jurisdictional claims in published maps and institutional affiliations.

Copyright: (c) 2021 by the authors. Licensee MDPI, Basel, Switzerland. This article is an open access article distributed under the terms and conditions of the Creative Commons Attribution (CC BY) license (https:// creativecommons.org/licenses/by/ $4.0 /)$.

\begin{abstract}
In this study, the blade shape of the squirrel-cage fan system inside the range hood was optimized using the surrogate model to improve the maximum volume flow rate. The influence of computational fluid dynamics (CFD) noise was concerned. The regression Kriging model (RKM) was used as a surrogate model to reflect the relationship between the design parameters of the blade and the volume flow rate. The parallel filling criterion after re-interpolation was used to improve the optimization efficiency further and ensure global optimization. Through experimental verification, we found that the relative error between the volume flow rate of the optimal sample of RKM and the experiment was only $0.4 \%$. Compared with the prototype, the maximum volume flow rate of the optimal sample of RKM was increased by $2.9 \%$, and the efficiency under the corresponding working conditions was increased by $2 \%$. RKM was used to predict the velocity field of the volute and impeller exit section to explore the feasibility of the RKM in the flow field prediction. Research shows that the RKM cannot accurately predict the velocity of each grid on the cross-section. Still, it can accurately predict the changing trend of the velocity.
\end{abstract}

Keywords: squirrel-cage fan; computational fluid dynamics (CFD); surrogate model; parallel filling criterion; flow field prediction

\section{Introduction}

The range hood is almost a necessary product in the kitchen to reduce the harm of lampblack to human beings. The squirrel-cage fan system mainly undertakes the internal ventilation function. The typical characteristics of a squirrel-cage fan are a large blade exit angle (strong forward blade), many blades (up to 60 pieces), and a short blade path. There are severe flow separation [1] and cross impeller flow in the fan [2]. As well as the complicated geometry of the range hood and the harsh air intake conditions of the fan, the highest efficiency of the range hood is less than $50 \%$. In developing range hood, aerodynamic analysis and optimization methods based on highly reliable computational fluid dynamics (CFD) are often used to improve the volume flow and efficiency. Due to the complexity of the structural design of the range hood, a large number of grid and computing resources are needed to obtain the reliable three-dimensional flow inside the squirrel-cage fan system. Under the background of exploiting the range hood's potential improvement, the way in which to solve the contradiction between the CFD-based method to obtain the optimal design parameters and computing resources is one of the most critical problems.

In the specific engineering application of obtaining optimal design parameters based on CFD, an optimization method based on the surrogate model is widely used to solve many calculations and time-consuming problems. The surrogate model is a mathematical model that can replace the complex and time-consuming numerical analysis model in 
optimization design [3]. The more common surrogate models in engineering applications include the Kriging model [4], neural network [5,6] and support vector regression [7,8], among others. For this approximate replacement surrogate model, the optimization result largely depends on the sample set and the approximate accuracy of the surrogate model. As the surrogate model is widely studied in the aerospace field, it has been developed to use various filling criteria to continuously add new sample points to retrain the surrogate model to ensure that it converges to the optimal global solution [9-11]. The most direct filling criterion is the minimizing surrogate predictor (MSP) criterion [12]. Other standard filling criteria include the expected improvement (EI) criterion $[13,14]$, the probability of improvement (PI) criterion [15,16], and the lower confidence bound (LCB) criterion [17], among others. Liu et al. [18] systematically compared the similarities and differences of various filling criteria, finding that the parallel filling can overcome the shortcomings of a single filling criterion and can improve the optimization efficiency and the robustness of the surrogate model at the same time.

Most of the existing filling criteria are based on the Kriging model in specific engineering applications, without considering the error between CFD and experiment. The EI and PI criteria are based on the basic assumption that the engineering function to be fitted is continuous and smooth [3]. The CFD noise will destroy the continuity and smoothness of the engineering function, thereby affecting the convergence and accuracy of the parallel filling criterion. Although the regression Kriging model [19] has long existed to solve this problem, the parallel filling criterion of the regression Kriging model is rarely applied to specific engineering applications. Its convergence and the validity of the parallel filling criterion are worthy of further study. For this reason, this study used the parallel filling method of EI, PI, and MSP to explore the role of the regression Kriging model in the performance optimization of range hoods.

In the rapid development of artificial intelligence, the coupling of CFD and machine learning methods based on many samples has become one of the research hotspots in flow field prediction and flow field control. On the basis of CFD and experiments, Bonakdari et al. [20] used an artificial neural network (ANN) to perform numerical analysis and prediction of the flow field in a $90^{\circ}$ elbow. The results show that the two training methods have a good approximation to predicting the curve speed field. Raissi et al. [21] propose a hidden fluid mechanics algorithm to complete the visualization of flow by learning velocity and pressure field. In terms of flow field control, Rabault et al. [22] first proposed the application of artificial neural network training and a deep reinforcement learning model to perform active flow control in a two-dimensional cylindrical flow. Li et al. [23] used machine learning to detect the turbulent area in the airflow flowing through a threedimensional cylinder. The common point of the above studies is that a large number of samples are required for training to obtain an accurate machine learning model. Still, it is difficult to obtain such a large amount of training data in complex practical engineering applications such as range hoods. This study further used the samples of the surrogate model established in the optimization process to verify the feasibility of the surrogate model in the prediction of the flow field of the range hood. The regression Kriging model was used to complete the flow field prediction of the critical cross-section inside the squirrel-cage fan with a small sample.

Figure 1 shows the research route of this article, which mainly includes three parts. In the first part, the aerodynamic performance of the whole range hood was tested to obtain the performance curve. The entire range hood was modeled, meshed and the flow field was solved, and the grid independence was verified to obtain a reliable grid with fewer grids. In the second part, the blade of the squirrel-cage fan was parameterized to obtain the three design parameters: blade outlet angle $\beta_{2}$, maximum bending of blade camber line $f$, and change of blade outlet position $\Delta \theta$. The range of design parameters was obtained according to the design experience. The optimal Latin hypercube design samples with nine samples were generated as the initial samples. According to the initial samples and the subsequent parallel filling criteria, we continuously trained the regression Kriging 
model for predicting the range hood's performance until the convergence condition was reached. Finally, the genetic algorithm was used to solve the optimal design parameters, and experimental verification was carried out. In the third part, on the basis of the samples in the optimization process, the regression Kriging model was used to predict the flow field of the critical sections in the squirrel-cage fan system. The prediction results were analyzed to find the internal reasons why the regression Kriging model can accurately predict the performance.

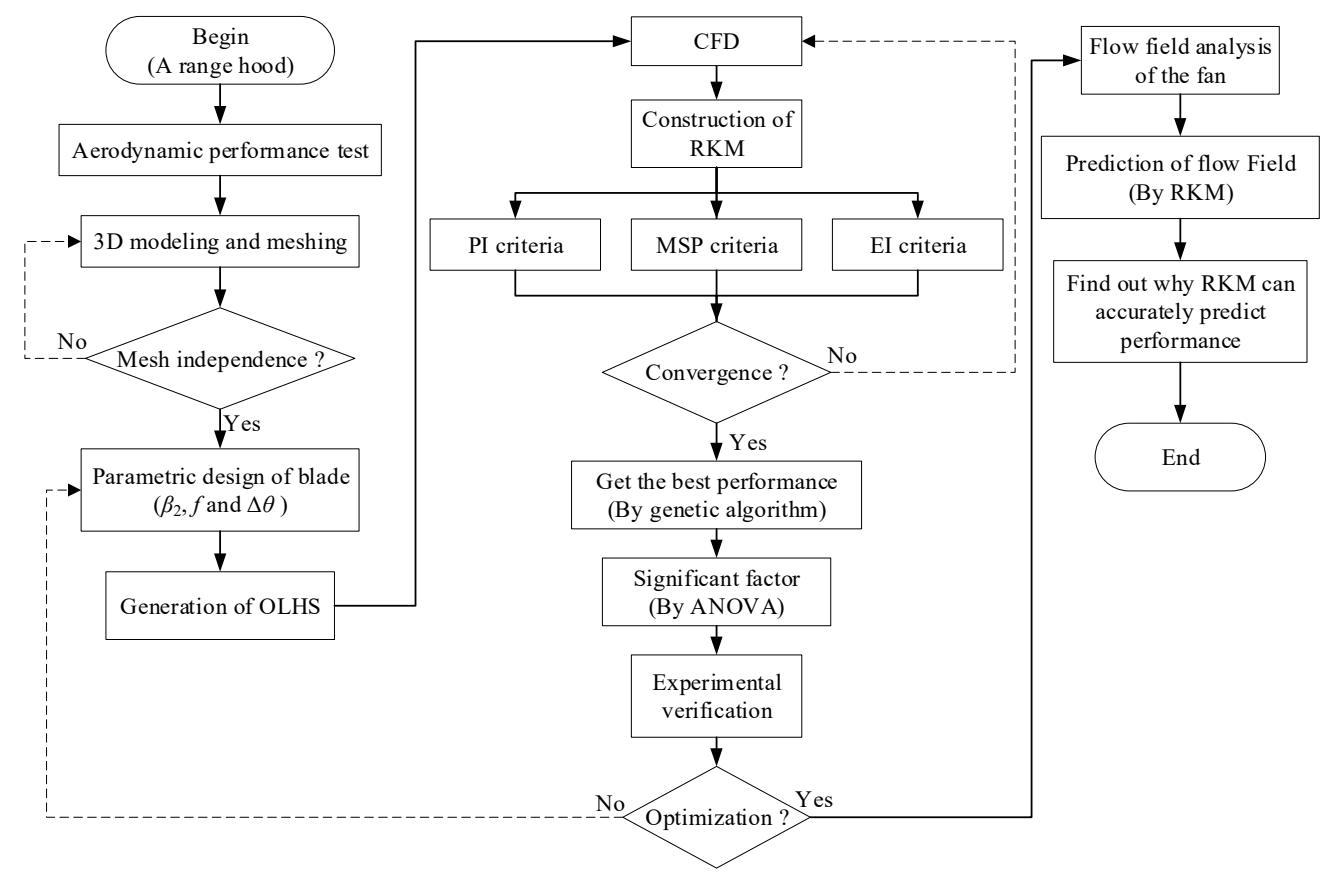

Figure 1. Research route.

\section{Research Object}

2.1. Geometric Model

Figure 2 shows a schematic diagram of the structure of a specific range hood. The lampblack flows in from the air intake cavity in a gathered manner and after passing through the grille. The lampblack is rectified by the collector and enters the inside of the fan. The kinetic energy of the lampblack increases when it works through the impeller. The high-speed lampblack flowing from the impeller flows into the volute. Its kinetic energy is converted into static pressure and flows to the smoke exhaust ducts through the outlet collector.

Table 1 and Figure 3 show the geometric parameters and schematic diagram of the fan, respectively. The sheet metal parts used in the shape of the entire impeller ensure that the blades are of equal thickness. Under this condition, the shape of the blade depends on the form of the camber line. The enlarged part in the red box in Figure 3 is the prototype's blade camber line, a single arc. The fan is the core component of the range hood. The impeller is the only rotating part of the fan, and its shape greatly influences the internal flow field and performance of the squirrel-cage fan. Yang et al. [24] used more complex double-arc blades to replace the commonly used single-arc blades. It was found that compared with single-arc blades with the same inlet and outlet angles, double-arc blades have higher fan pressure and harm efficiency. Wen et al. [25] used blade trimming to improve the fan's internal flow state and performance. Therefore, the optimization of blade geometry is one of the most effective ways to improve fan performance. 


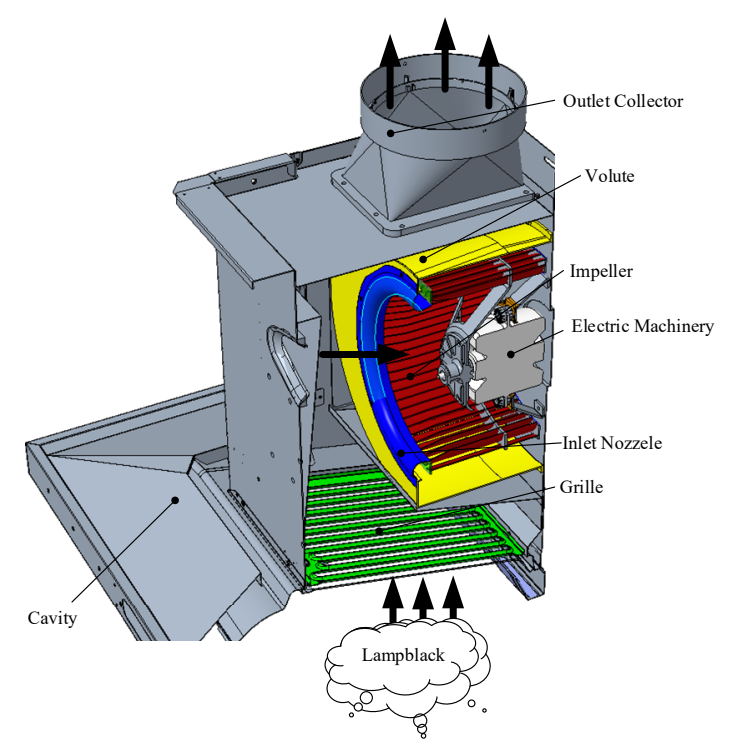

Figure 2. Structure diagram of a range hood.

Table 1. Geometrical parameters of the squirrel-cage fan.

\begin{tabular}{cc}
\hline Geometrical Parameters & Size \\
\hline Impeller width, $b_{I}(\mathrm{~mm})$ & 146.4 \\
Impeller inlet diameter, $D_{1}(\mathrm{~mm})$ & 210 \\
Impeller outlet diameter, $D_{2}(\mathrm{~mm})$ & 250 \\
Blade inlet angle, $\beta_{1}(\mathrm{deg})$ & 85 \\
Blade outlet angle, $\beta_{2}(\mathrm{deg})$ & 177 \\
Number of blades, $Z$ & 60 \\
Volute tongue radius, $R_{V}(\mathrm{~mm})$ & 8.9 \\
Impeller-tongue clearance, $\mathrm{d}_{t}(\mathrm{~mm})$ & 24 \\
Volute width, $b_{V}(\mathrm{~mm})$ & 173 \\
Chord length of midline of blade, $b(\mathrm{~mm})$ & 27.7 \\
Maximum camber, $f(\mathrm{~mm})$ & 6.5 \\
Blade thickness, $d(\mathrm{~mm})$ & 1.2 \\
\hline
\end{tabular}

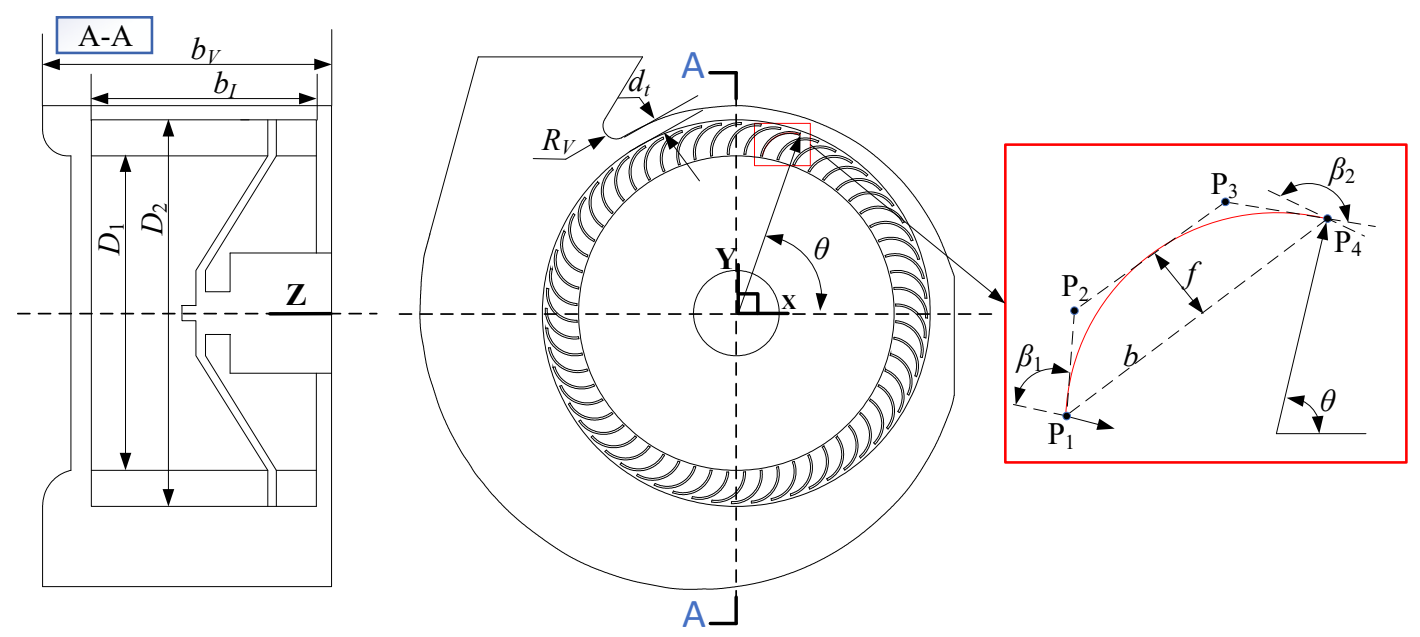

Figure 3. Geometric parameters of squirrel-cage fan.

Compared with a single arc, the spline curve has a more flexible and complex shape, one that is widely used in fan blade design [26]. Concerning the parametric description of the blades in the axial flow fan [27], we used the B-spline curve to control the shape of the blades. In blade parameterization, the center of the impeller rotation axis was taken as the 
origin of the Cartesian coordinate system. The length of the endpoint $\mathrm{P}_{1}$ and $\mathrm{P}_{4}$ at both ends of the camber line is the chord length $\mathrm{b}$. The angle between the endpoint $\mathrm{P}_{4}$ and the origin and the $x$-axis is the angle $\theta$. Since the impeller outlet diameter remains unchanged, angle $\theta$ determines the position of endpoint $\mathrm{P}_{4}$. The distance $f$ between line segment $\mathrm{P}_{1} \mathrm{P}_{4}$ and line segment $P_{2} P_{3}$ is the maximum bending of the camber line. $\beta_{1}$ and $\beta_{2}$ are the inlet angle and outlet angle of the blade, respectively. $\mathrm{P}_{1}, \mathrm{P}_{2}, \mathrm{P}_{3}$, and $\mathrm{P}_{4}$ are the control points of the quadratic non-uniform B-spline curve. When the blade exit angle $\beta_{2}$ is changed, the blade will exit position $\theta$, and the maximum curvature $f$ of the camber line will be in order, with the other parameters kept unchanged. The four control points $\mathrm{P}_{1}, \mathrm{P}_{2}, \mathrm{P}_{3}$, and $\mathrm{P}_{4}$ can be uniquely determined. The B-spline curve can be uniquely generated and used as the camber line of the blade from these four control points.

The camber line of the prototype was used as the reference, and the change $\Delta \theta$ of the blade exit position $\theta$ was used as the design variable. The blade parameterization method of determining a spline curve according to three specific blade parameters can avoid the appearance of strange blade shapes due to unreasonable design parameters. It ensures the continuity and smoothness of the engineering function to be fitted. Under the specific of certain design experiences, the range of design variables given in this paper is shown in Table 2.

Table 2. Variation range of design variables.

\begin{tabular}{cccc}
\hline Design Variable & Baseline & Lower Limit & Upper Limit \\
\hline$\beta_{2},{ }^{\circ}$ & 177 & 160 & 177 \\
$f, \mathrm{~mm}$ & 6.5 & 5 & 8 \\
$\Delta \theta^{\circ}$ & 0 & -3 & 3 \\
\hline
\end{tabular}

\subsection{Experimental Device}

Figure 4 shows the aerodynamic performance experimental device of the range hood. According to the international standard ISO5801-2007: Industrial fans, an experimental platform for standardized air passage performance test, we tested range hood performance in the air passage of the same standard. The performance curve was drawn on the basis of the data measured by the orifice method. Before the test, the current environment parameters such as pressure and temperature need to be input into the test program. The performance parameters of the range hood system were measured by replacing the hole board with varying sizes of the pore at the throttling device. The speed of the motor was measured by a stroboscope. The average value of the measured pressure of the four pressure taps evenly distributed on the pipe was taken as the static pressure $\Delta p$ after the airflow passes through the cell straightener. The volume flow rate $Q_{v}$, total pressure rise $P_{t F}$, total pressure efficiency $\eta$, and static pressure rise $P_{S F}$ of the range hood were calculated on the basis of the test data of the instrument by the following equation:

$$
\left\{\begin{array}{l}
Q_{v}=\frac{\pi}{4} \alpha_{7} d_{7}^{2} \sqrt{\frac{2 \Delta p_{6}}{\rho}} \\
\eta=\frac{Q_{v} \cdot P_{t F}}{W} \times 100 \% \\
P_{t F}=\Delta p+0.5 k\left(\frac{Q_{v}}{A_{2}}\right)^{2} \\
P_{S F}=P_{t F}-0.5 \rho\left(\frac{Q_{v}}{A_{2}}\right)^{2}
\end{array}\right.
$$

where $\Delta p_{6}$ is the average static pressure obtained by the pressure taps; $\rho$ is air density; $\alpha_{7}$ and $d_{7}$ are the coefficients and diameter of the hole board throttling device, respectively; $A$ is the area of the pipe connector; $k$ is the area ratio between sections A and $\mathrm{B}$; and $W$ is the $\mathrm{AC}$ motor input power. 

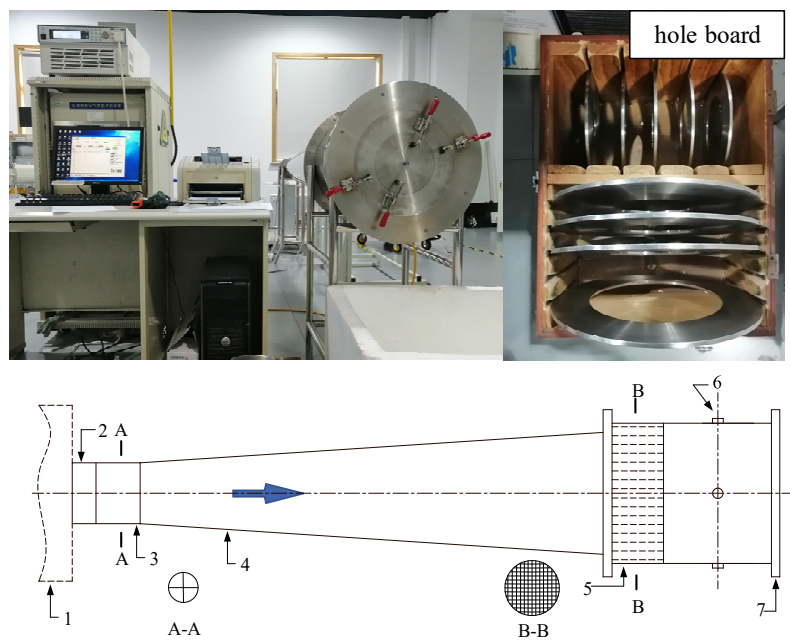

Figure 4. The aerodynamic performance experimental device of the range hood: (1) range hood; (2) pipe connector; (3) cross straightener; (4) diffusion channel; (5) cell straightener; (6) pressure taps; (7) hole board throttling device.

Before the test, the coefficient in the formula was calibrated, which can accurately obtain the actual performance of the range hood. The performance parameters of the range hood under 11 operating conditions were tested and fitted to the performance curve of the range hood. Figure 5 shows the changes of motor speed $n$, total pressure rise $P_{t F}$, static pressure rise $P_{S F}$, efficiency $\eta$, and power $W$ with volume flow rate $Q_{v}$.

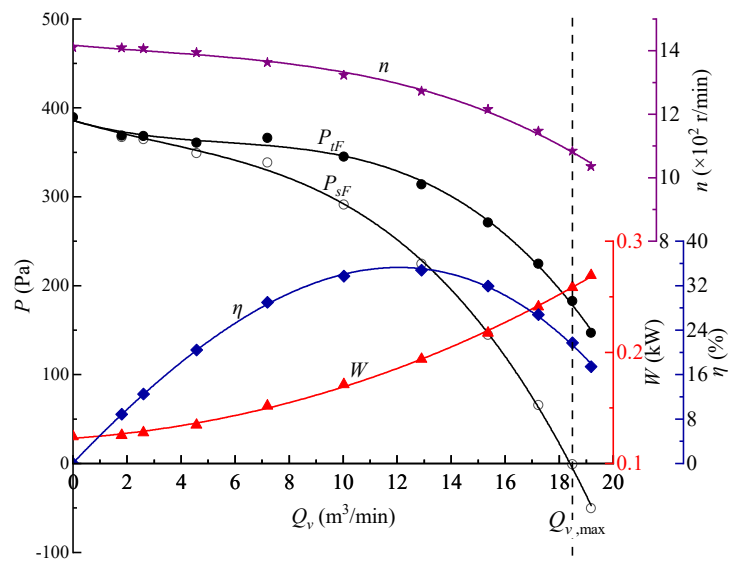

(a) Performance curve of range hood

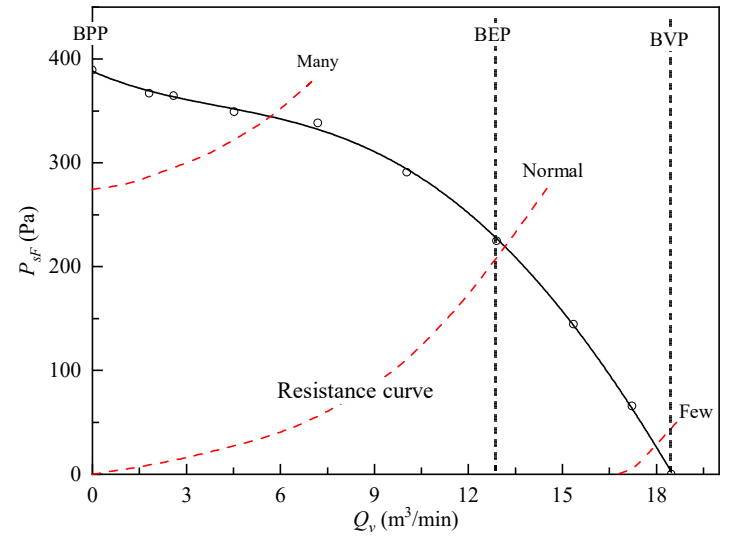

(b) The operating state of the range hood in the sharing smoke exhaust ducts

Figure 5. Operating status of range hood.

For residential buildings sharing smoke exhaust ducts, with the increasing number of range hoods used simultaneously, smoke exhaust ducts become increasingly crowded. As a result, the pressure in the exhaust duct increases. The operation condition of the range hood progresses to the state of decreasing volume flow rate, which means that less and less lampblack is discharged in unit time, and the smoke exhaust rate is reduced. There are three operating conditions to evaluate the performance of the range hood, which are the best volume flow rate point (BVP), the best efficiency point (BEP), and the best pressure point (BPP). The volumetric flow rate corresponding to BVP reflects the maximum smoke exhaust rate, which is the working condition with the highest smoke exhaust rate of the range hood. The corresponding efficiency of BEP is the operating design point where the range hood discharges the lampblack from the standard exhaust duct. The range hood generally runs near this operating point. The pressure corresponding to BPP is the ultimate 
pressure of the range hood exhaust, reflecting the maximum exhaust capacity of the range hood. This research focuses on the operating state of the range hood under BVP and optimizes the impeller of the fan system, intending to increase the volume flow rate.

\section{Numerical Methods}

Figure 6a shows the computation zone of the range hood, which is composed of four parts of the fluid area: cavity, volute, impeller, and outlet collector. The inlet and outlet of the range hood are appropriately extended to provide stable inflow and outflow conditions. The extension lengths of the inlet section of the cavity and the outlet section of the collector are one and four times the outer diameter of the impeller, respectively.

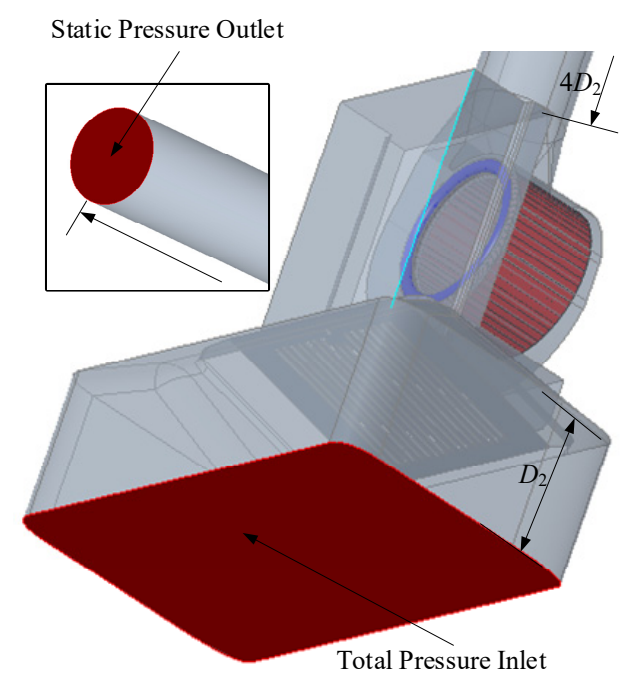

(a) Computation zone

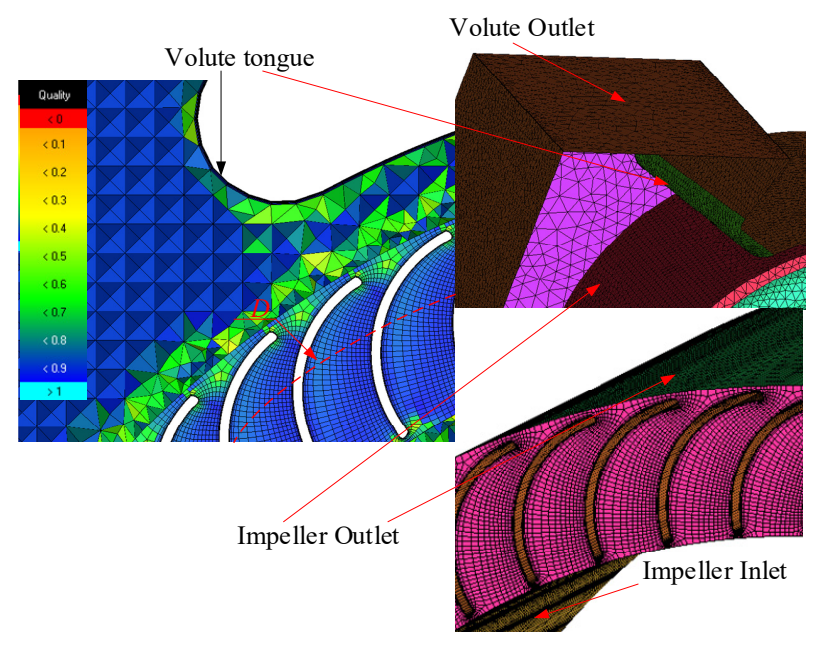

(b) Details of grid division

Figure 6. Computation zone and details of grid division.

The Ansys Turbogrid was used for the hexahedral structured meshing of the impeller computation zone. The ICEM was used for the unstructured tetrahedral meshing of the remaining computation zone. The mesh of each computation zones was finally combined in ICEM. In the process of meshing, the mesh of grille, AC motors, and other structures are encrypted, and the wall mesh of volutes and impellers are refined, and boundary layers mesh are added. The mesh details of the volute and the impeller are shown in Figure $6 \mathrm{~b}$.

Fluent was used to solve the three-dimensional flow field of range hoods. The Mach number of squirrel-cage fan system is less than 0.3 , which is regarded as incompressible [2]. The range hood contains a squirrel-cage fan system and no other rotating parts, which the airflow in the range hood can still be treated as incompressible. The shear stress transport (SST) $k$ - $\omega$ model [28] was used to calculate the turbulent viscosity. The boundary conditions of the inlet and outlet were set as pressure inlet and pressure outlet, respectively. Due to the extension section of the inlet and outlet, the inlet airflow and outlet airflow were fully developed. There was no backflow; the pressure direction was perpendicular to the boundary; and the turbulence intensity and turbulence viscosity ratio adopted the default turbulence flow, $5 \%$, and $10 \%$, respectively. The size of the pressure value corresponds to the total inlet pressure and outlet static pressure values under BVP conditions. The gauge total pressure of the pressure inlet and the gauge pressure of the pressure outlet were both set to zero. The multiple reference frame (MRF) was employed to deal with the rotating impeller computation zone. The coincidence surface between the flow zones was set to mesh interface to facilitate the data transfer between the flow zones. The SIMPLE algorithm was used to realize the coupling of velocity and pressure. Second-order upwind discretization was used to disperse turbulent dissipation, momentum, and turbulent kinetic energy. 
The amount of mesh for range hoods was increased from 3 million to 12 million to verify the independence of the mesh. The amount of mesh in each computation zone was adjusted to achieve a uniform increase in the mesh by changing the global factor of mesh. Taking the volume flow rate $Q_{v}$ under BVP conditions as the evaluation index, Figure 7 shows the error between the calculated results of different mesh amounts and the experimental values. As the amount of mesh increased, the error continued to decrease. When the number of grids was 7.2 million, the error can be controlled within $4 \%$. However, as the amount of mesh continued to increase, there was no significant improvement in the prediction accuracy of the volume flow rate. The computing power and prediction accuracy were considered, and the grid division model corresponding to 9.3 million was finally determined as the mesh model for subsequent optimization. The number of grids corresponding to each zone is shown in Table 3.

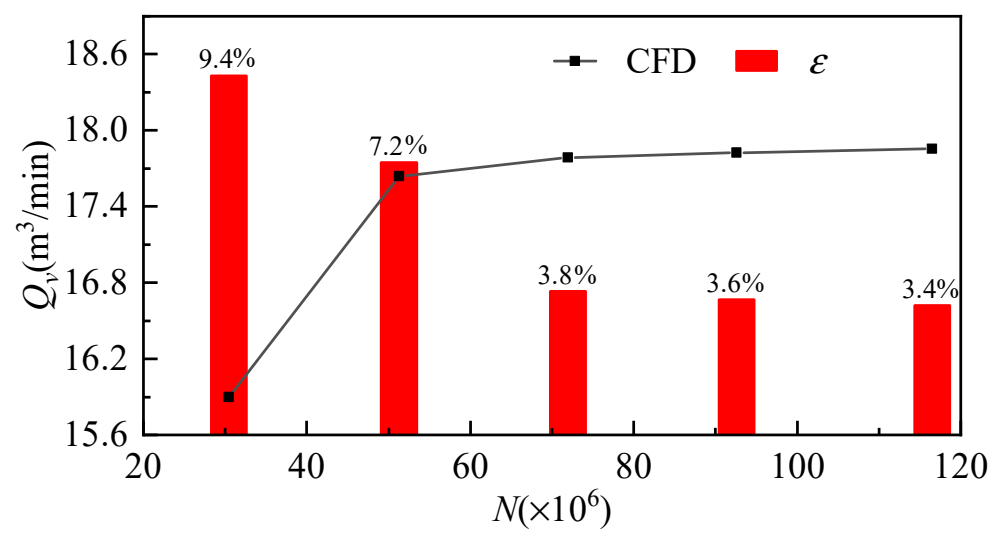

Figure 7. Independent grid validation.

Table 3. Grid quantity (unit: million).

\begin{tabular}{cccccc}
\hline Flow Zones & Cavity & Volute & Impeller & $\begin{array}{c}\text { Outlet } \\
\text { Collector }\end{array}$ & Total \\
\hline Quantity & 2.6 & 3.3 & 3 & 0.4 & 9.3 \\
\hline
\end{tabular}

We set the gauge pressure at the pressure outlet and the speed of the impeller computation zone to the experimental static pressure rise and speed under other working conditions, respectively. The comparison between the predicted volume flow rate $Q_{v}$ and efficiency $\eta$ and the experimental value is shown in Figure 8. The maximum error of $Q_{v}$ was less than $4 \%$ between BVP and BEP, and the trend between predicted efficiency and experimental value was consistent. Therefore, the numerical method used in this study is reliable under the condition of a large volume flow rate. However, with the further decrease of $Q_{v}$, the prediction error after the BEP condition increased rapidly. The error between the performance prediction of the range hood and the experimental value is inevitable, mainly caused by two reasons. The first reason is that the geometric structure of the range hood mesh model has many simplifications and inherent errors, such as CFD and experimental tests. The second reason is that the blade deformation caused by the high-speed rotating sheet metal impeller is not considered. Especially in the smaller volume flow rate condition, the impeller rotation speed is higher, and the blade with a thickness of only $0.4 \mathrm{~mm}$ is more prone to deformation. 


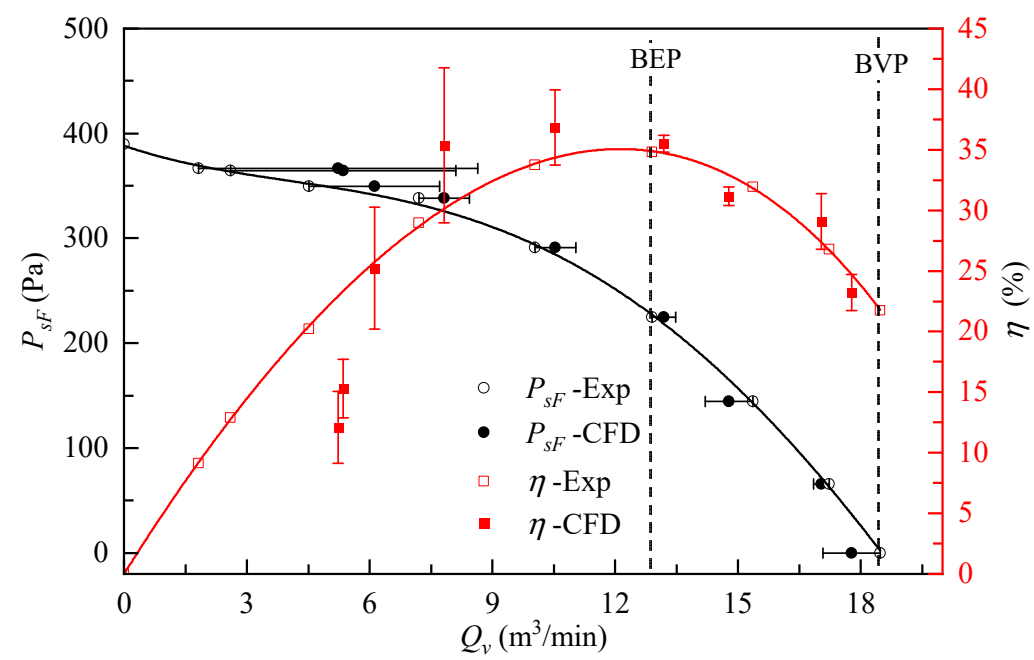

Figure 8. Comparison of the CFD and experimental.

\section{Optimization}

\subsection{Space-Filling Latin Hypercubes}

The first step of surrogate model optimization is to generate a set of initial sample points and establish an initial surrogate model according to the range of design parameters (Table 2) and design of experiments (DOE) technique. The idea of DOE is to maximize the information of unknown space by selecting a small number of sample points. Giunta et al. [29] divided DOE into classical and modern DOE. The classical DOE, such as central composite design, Box-Behnken design, and full- and fractional-factorial design, are mainly used to arrange the instrument experiment and consider reducing the influence of random error. The modern DOE, such as the Monte Carlo method, Latin hypercube sampling (LHS), and orthogonal experimental design, mainly adopt space-filling to arrange computer experiments. The LHS divide the design space into many hypercubes of the same size and place sample points in these hypercubes in the form of only one sample per row and column. The LHS can accurately extract trend information and is widely used in surrogate model optimization.

However, the Latin hypercube is random, and there is no guarantee that the generated samples are evenly filled in the entire design variable space. For example, placing all sample points on the main diagonal of the design space can meet the standard, but it does not fill the entire space uniformly. Morris and Michell [30] proposed a scalar-valued design criterion to measure the space-filling ability of LHS:

$$
\Phi_{p}(D)=\left(\sum_{j=1}^{m} J_{j} d_{j}^{-p}\right)^{1 / p}
$$

where $p$ and $m$ are a positive integer and number of samples, respectively. According to the suggestions of Morris and Mitchell [30], this study takes the smallest $\Phi_{p}$ among $p=1$, $2,5,10,20,50,100 . d_{1}, d_{2}, \ldots, \mathrm{dm}$ are the sequence in which the distances between the two sample points of all possible combinations in the sampling matrix $D$ are arranged in ascending order. $J_{1}, J_{2}, \ldots, J_{m}$ are the number of distances $d_{j}$ between two points in the sample matrix $D$. When the value of $\Phi_{p}$ is smaller, the space-filling of $D$ is better. The distance $d$ between the two sample points $x_{s}$ and $x_{t}$ is defined by

$$
d\left(x_{s}, x_{t}\right)=\left(\sum_{l=1}^{k}\left|x_{s}{ }^{(l)}-x_{t}{ }^{(l)}\right|^{q}\right)^{\frac{1}{q}}
$$


where $k$ is the dimension of the sample point, and $q$ is a positive integer, indicating the type of distance. Since the calculation of the rectangular distance $(q=1)$ is less than the Euclidean distance $(q=2)$, the rectangular distance was used in this study.

Forrester and Keane [31] pointed out that the number of initial sample points of the surrogate model is theoretically not limited by the dimension of the design space, and the optimization efficiency does not depend significantly on the number of initial sample points. Since this research aims to obtain the optimal global solution, many new samples should be added through the parallel filling criterion. Sóbester [32] recommended that the ratio of the initial number of sample points to the number of sample points added in the later period is about 1:2. In this study, with only three design parameters, the initial space-filling LHS (Table 4) with nine samples was generated according to variable range (Table 2).

Table 4. Initial space-filling LHS.

\begin{tabular}{ccccc}
\hline No. & $\boldsymbol{B}_{\mathbf{2}}$ & $f$ & $\boldsymbol{\Delta} \boldsymbol{\theta}$ & $\boldsymbol{Q}_{\boldsymbol{v}}$ \\
\hline 1 & 168.50 & 5 & 1.5 & 18.41 \\
2 & 172.75 & 5.38 & -2.25 & 17.52 \\
3 & 166.38 & 6.88 & -3 & 17.5 \\
4 & 174.88 & 7.63 & -1.5 & 15.16 \\
5 & 170.63 & 7.25 & 2.25 & 18.37 \\
6 & 160 & 5.75 & -0.75 & 18.28 \\
7 & 162.13 & 6.5 & 3 & 18.78 \\
8 & 177 & 6.13 & 0.75 & 17.84 \\
9 & 164.25 & 8 & 0 & 17.5 \\
\hline
\end{tabular}

Figure 9 shows the specific distribution of the initial sample points in the space. The red dot represents the initial sample point, and the black dot represents the projection of the sample point on the coordinate system. The projection of sample points that the distribution of sample points is relatively uniform, and the projection of some sample points is distributed on the boundary, which is conducive to enhancing the fitting ability of the surrogate model in the whole design space.

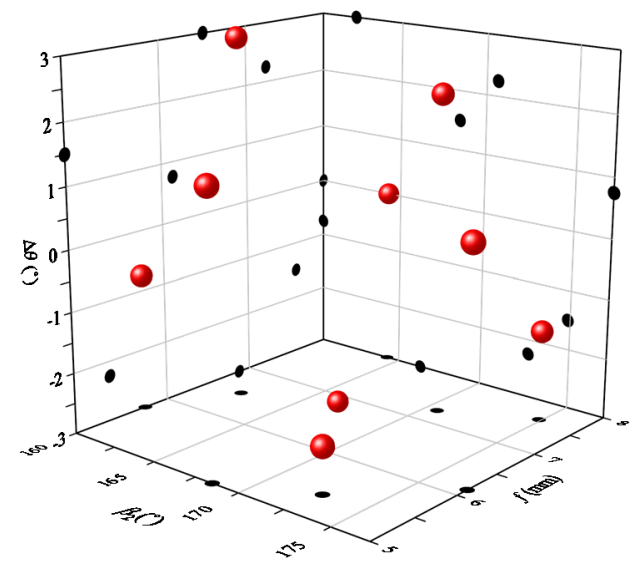

Figure 9. Three-dimensional diagram of initial sample points.

\subsection{Parallel Filling Criterion of RKM}

The choice of the type of filling criteria has a greater impact on the global search performance of the surrogate model. Due to the poor performance of the MSE filling criteria [14] and the complexity of the LCB filling criteria corresponding to RKM, this research only conducted applied research on the parallel filling criteria of MSP, PI, and EI. 


\subsubsection{Minimizing Surrogate Predictor}

The prediction $\hat{y}$ obtained by the surrogate model is an approximation of the real $y$. The simplest and most direct filling criterion is to add the optimal solution of the current model as a new sample to the sample space to improve the model's prediction accuracy near the optimal solution. Jones et al. [14] pointed out that MSP is a local mining method and cannot have the ability to find the optimal global solution. Since this research solves the maximum value, the corresponding MSP filling criterion is

$$
\begin{gathered}
M S P=\operatorname{Min}[-\hat{y}(x)] \\
\text { s.t. }\left\{\begin{array}{c}
160 \leq \beta_{2} \leq 177 \\
5 \leq f \leq 8 \\
-3 \leq \Delta \theta \leq 3
\end{array}\right.
\end{gathered}
$$

\subsubsection{Probability of Improvement}

The MSP filling criterion is common to any surrogate model. Still, the surrogate model based on the Gauss process, such as the Kriging model, can regard the prediction $\hat{y}$ as a specific realization of a random process $Y \sim N\left[\hat{y}(x), s^{2}(x)\right]$. When searching for the location of the next sample point, find the point $x=\left(\beta_{2}, f, \Delta \theta\right)$ with the greatest probability of improving the optimal observation value $y_{\min }$ of the current surrogate model, and use this point as the location of the next update point. According to the estimated mean square error (MSE) of the prediction $\hat{y}$, the probability $P[I(x)]$ of the improvement $I(x)=y_{\min }-Y(x)$ on $y_{\min }$ can be obtained:

$$
P[I(x)]=\frac{1}{s(x) \sqrt{2 \pi}} \int_{-\infty}^{0} e^{-[I-\widehat{y}(x)]^{2} /\left(2 s^{2}\right)} d I=\frac{1}{2}\left[1+\operatorname{erf}\left(\frac{y_{\min }-\widehat{y}(x)}{s(x) \sqrt{2}}\right)\right]
$$

where $\hat{y}(x)$ and $s(x)$ are the mean and variance of the random process $I(x)$, respectively. $s(x)$ also represents the estimated MSE in the surrogate model.

\subsubsection{Expected Improvement}

Given the mean $\hat{y}(x)$ and variance $s(x)$, in addition to calculating $P[I(x)]$, it can also calculate the expected value of improvement $E[I(x)]$ :

$$
\begin{aligned}
& E[I(x)]=\left\{\begin{array}{c}
\left(y_{\min }-\hat{y}(x)\right) \Phi\left(\frac{y_{\min }-\hat{y}(x)}{s^{2}(x)}\right) \\
+s(x) \phi\left(\frac{y_{\min }-\hat{y}(x)}{s^{2}(x)}\right), s>0 \\
0, s=0
\end{array}=\right. \\
& \left(y_{\min }-\hat{y}(x)\right)\left[\frac{1}{2}+\frac{1}{2} \operatorname{erf}\left(\frac{y_{\min }-\hat{y}(x)}{s(x) \sqrt{2}}\right)\right]+s(x) \frac{1}{\sqrt{2}} \exp \left[\frac{-\left(y_{\min }-\hat{y}(x)\right)^{2}}{2 s^{2}(x)}\right]
\end{aligned}
$$

where $\boldsymbol{\Phi}(\cdot)$ and $\phi(\cdot)$ are the cumulative distribution and probability density function of standard normal distribution, respectively.

Forrester and Keane [3] proved that a new sample point with the maximum EI and PI under the current model could obtain the optimal global solution of the engineering function. Still, these two criteria are based on the basic assumption that the engineering function to be fitted is continuous and smooth. However, CFD-based computer simulation will cause a certain error between the function value and the real value, which means that the predicted value will scatter around the real function. The CFD noise will destroy the smoothness, which is not convenient for the optimization search with additional criteria. RKM adds a regression coefficient $\lambda$ to the diagonal of the correlation matrix to ensure the smoothness of the engineering function and meet the assumptions of EI and PI filling criteria. Following the method of deriving the Kriging model, the expression of the prediction $\hat{y}_{r}$ and estimated MSE $\hat{s}_{r}$ of the RKM can be obtained as

$$
\hat{y}_{r}=\hat{\mu}+\boldsymbol{\varphi}^{\mathrm{T}}(\boldsymbol{\psi}+\lambda \mathbf{I})^{-1}(y-1 \hat{\mu})
$$




$$
\hat{s}_{r}{ }^{2}(x)=\hat{\sigma}_{r}\left[\mathbf{1}+\lambda-\boldsymbol{\varphi}^{\mathrm{T}}(\boldsymbol{\psi}+\lambda \mathbf{I})^{-1} \boldsymbol{\varphi}+\frac{\mathbf{1}-\mathbf{1}^{\mathrm{T}}(\boldsymbol{\psi}+\lambda \mathbf{I})^{-1} \boldsymbol{\varphi}}{\mathbf{1}^{\mathrm{T}}(\boldsymbol{\psi}+\lambda \mathbf{I})^{-1} 1}\right]
$$

where $\lambda$ is the regression coefficient. $\mathbf{1}$ and $\mathbf{I}$ are the unit column vector of order $n \times 1$ and the unit matrix of order $n \times n$, respectively. $\psi$ and $\varphi$ are the $n \times n$ order correlation matrix of all sample points and the $n \times 1$ order correlation matrix of the sample and the predicted sample, respectively. Obtain the mean and variance of the RKM based on maximum likelihood estimation:

$$
\begin{gathered}
\hat{\mu}=\frac{\mathbf{1}^{\mathrm{T}}(\boldsymbol{\psi}+\lambda \mathbf{I})^{-1} y}{\mathbf{1}^{\mathrm{T}}(\boldsymbol{\psi}+\lambda \mathbf{I})^{-1} \mathbf{1}} \\
\hat{\sigma}_{r}{ }^{2}=\frac{(y-\mathbf{1} \hat{\mu})^{\mathrm{T}}(\boldsymbol{\psi}+\lambda \mathbf{I})^{-1}(y-\mathbf{1} \hat{\mu})}{n}
\end{gathered}
$$

In the process of optimizing the search on the basis of the PI and EI criterion, the predicted value of RKM will not strictly pass the initial sample point due to the influence of the regression coefficient. It means that the PI and EI at the initial sample point are not zero to produce the improvement effect at the initial sample point. Suppose the filling is performed according to the EI criterion and the PI criterion. In that case, the newly added sample points may overlap with the existing sample points, which will cause the optimization process to stagnate and fail to converge to the optimal solution.

The predicted value was re-interpolated [31] to make the EI and PI at the existing sample point both zero. The estimated MSE $\hat{s}_{r i}$ after re-interpolation is

$$
\hat{s}_{r i}{ }^{2}(x)=\hat{\sigma}_{r i}\left[\mathbf{1}-\boldsymbol{\varphi}^{\mathrm{T}} \boldsymbol{\psi}^{-1} \boldsymbol{\varphi}+\frac{1-\mathbf{1}^{\mathrm{T}} \boldsymbol{\psi}^{-1} \boldsymbol{\varphi}}{\mathbf{1}^{\mathrm{T}} \boldsymbol{\psi}^{-1} \mathbf{1}}\right]
$$

The variance after re-interpolation is

$$
\hat{\sigma}_{r i}=\frac{(y-\mathbf{1} \hat{\mu})^{\mathrm{T}}(\boldsymbol{\psi}+\lambda \mathbf{I})^{-1} \boldsymbol{\psi}(\boldsymbol{\psi}+\lambda \mathbf{I})^{-1}(y-\mathbf{1} \hat{\mu})}{n}
$$

The estimated MSE $\hat{s}_{r i}$ and variance after re-interpolation were substituted into Equations (5) and (6) to obtain the re-interpolated EI and PI filling criterion, thereby restoring the global convergence characteristics of the EI and PI filling criterion. In the subsequent optimization process, the parallel filling criterion of EI, PI, and MSP filling criterion was used to improve further the optimization efficiency of using RKM as a surrogate model.

\subsection{Optimization Stage}

The optimization process based on the surrogate model must have an optimization termination condition. However, the current parallel filling criterion based on the RKM does not have a clear optimization termination condition. According to the situation of subsequent filling, the optimization termination condition of this study is that the filling criterion of EI, PI, and MSP all reach convergence. For EI and PI filling criterion, when the maximum EI and PI reach a certain threshold, it can be considered to have converged.

$$
\left\{\begin{array}{l}
\mathrm{EI}_{\max } \leq 1 \times 10^{-6} \\
\mathrm{PI}_{\max } \leq 1 \times 10^{-6}
\end{array}\right.
$$

For the MSP filling criterion, when the difference between the newly added sample point and the optimal value of the last added point is less than a given threshold, it is considered to have converged.

$$
\Delta \mathrm{MSP}=\frac{\left|\mathrm{y}_{\text {new,best }}-\mathrm{y}_{\text {old,best }}\right|}{\left|\mathrm{y}_{\text {old,best }}\right|} \leq 1 \times 10^{-2}
$$


where $x_{\text {new,best }}$ and $y_{\text {new,best }}$ are the newly added sample points according to the MSP filling criterion and the optimal value predicted by RKM after the update. $x_{\text {old,best }}$ and $y_{\text {old,best }}$ are the sample points added last time according to the MSP criterion and the optimal value predicted by RKM.

Figure 10 shows the maximum EI, the maximum PI, and $\triangle \mathrm{MSP}$ variation with the number of parallel fillings. After the sixth time of parallel filling, EI, PI, and $\triangle \mathrm{MSP}$ were all less than the given threshold, satisfying the optimal termination condition. According to the changes of $\mathrm{EI}_{\max }, \mathrm{PI}_{\max }$, and $\triangle \mathrm{MSP}$ and the predictive ability of RKM in the parallel filling process, the parallel filling process in this study can be divided into an initial stage (0 to 1 parallel filling) and an improvement stage (2 to 6 parallel fillings) and the convergence stage (7 to 9 parallel fillings).

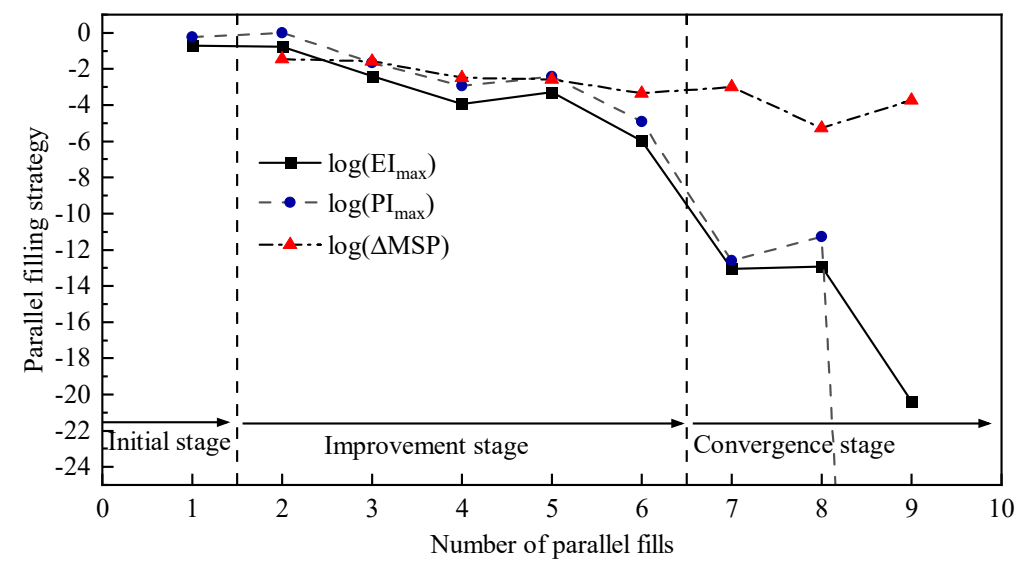

Figure 10. Parallel filling process.

\subsubsection{Initial Stage}

Figure 11 shows the prediction results of the sample points by RKM at the initial stage. Table 5 shows the detailed sample data, where the fill factor represents the maximum $\mathrm{EI}_{\max }$, $\mathrm{PI}_{\max }$, or MSP. As shown in Figure 11a, when the initial number of samples was only nine, the predicted values of the RKM and the Kriging model were the same, and the three new sample points obtained by parallel filling were also the same. Figure $11 \mathrm{~b}$ shows that when three new sample points were added to the sample space. At this time, the prediction results of the RKM and Kriging model are still highly consistent. The maximum relative error between the predicted value of the regression Kriging model and the CFD value was $0.32 \%$, which did not reflect the effect of the RKM in filtering noise after adding regression coefficients $\lambda$. The noise generated by CFD will be ignored by RKM when the number of samples is small.

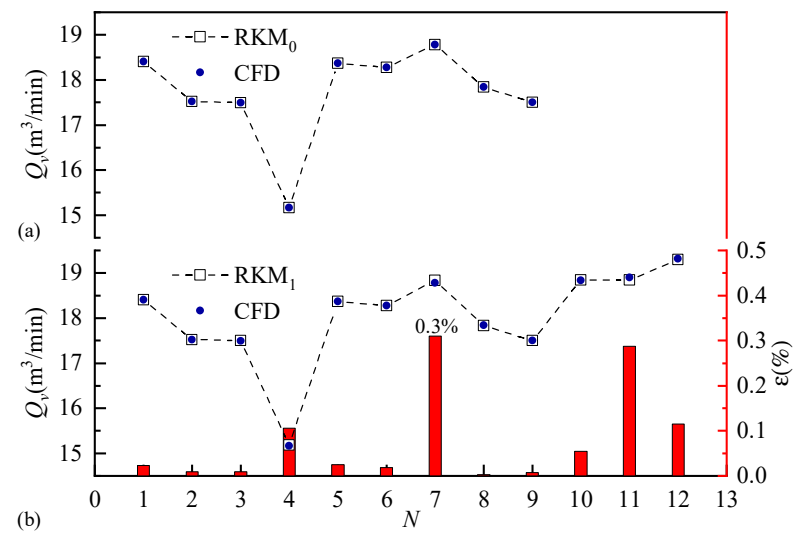

Figure 11. The prediction results of RKM for sample points in the initial stage: (a) initial sample; (b) sample after first filling. 
Table 5. Filling process of RKM in the initial stage.

\begin{tabular}{|c|c|c|c|c|c|c|c|c|}
\hline $\begin{array}{l}\text { Filling } \\
\text { Times }\end{array}$ & $\begin{array}{l}\text { Number of } \\
\text { Samples }\end{array}$ & $\begin{array}{l}\text { Filling } \\
\text { Criterion }\end{array}$ & $\begin{array}{l}\beta_{2} \\
\left(^{\circ}\right)\end{array}$ & $\underset{(\mathrm{mm})}{f}$ & $\begin{array}{l}\Delta \theta \\
\left({ }^{\circ}\right)\end{array}$ & Fill Factor & $\begin{array}{c}\text { Predictive } \\
\text { Value } \\
\left(\mathrm{m}^{3} / \mathrm{min}\right)\end{array}$ & $\begin{array}{c}\text { CFD } \\
\left(\mathrm{m}^{3} / \mathrm{min}\right)\end{array}$ \\
\hline \multirow{3}{*}{1} & 10 & EI & 160 & 6.43 & 1.69 & 0.2 & 18.67 & 18.84 \\
\hline & 11 & PI & 162.13 & 6.5 & 3 & 0.58 & 18.78 & 18.9 \\
\hline & 12 & MSP & 162.47 & 6.42 & 2.64 & 18.8 & 18.8 & 19.32 \\
\hline
\end{tabular}

\subsubsection{Improvement Stage}

Table 6 shows the filling process in the improvement stage. When RKM was filled for the second time, the number of samples was 15. As shown in Figure 12a, at this time, the prediction results of the RKM and the filling position began to show obvious differences with the Kriging model. The maximum relative error between the predicted and the CFD was $2 \%$. From the second filling to the sixth filling, the maximum EI and PI rapidly decreased with the optimization process, indicating that the predictive ability of RKM is continuously improving.

Table 6. Filling process in the improvement stage.

\begin{tabular}{|c|c|c|c|c|c|c|c|c|}
\hline $\begin{array}{l}\text { Filling } \\
\text { Times }\end{array}$ & $\begin{array}{c}\text { Number of } \\
\text { Samples }\end{array}$ & $\begin{array}{l}\text { Filling } \\
\text { Criterion }\end{array}$ & $\begin{array}{l}\beta_{2} \\
\left({ }^{\circ}\right)\end{array}$ & $\underset{(\mathrm{mm})}{f}$ & $\begin{array}{l}\Delta \theta \\
\left({ }^{\circ}\right)\end{array}$ & Fill Factor & $\begin{array}{l}\text { Predictive } \\
\text { Value } \\
\left(\mathrm{m}^{3} / \mathrm{min}\right)\end{array}$ & $\begin{array}{c}\text { CFD } \\
\left(\mathrm{m}^{3} / \mathrm{min}\right)\end{array}$ \\
\hline \multirow{3}{*}{2} & 13 & EI & 160.23 & 6 & 2.39 & 0.17 & 19.39 & 18.77 \\
\hline & 14 & PI & 162.29 & 6.4 & 2.56 & 0.96 & 19.37 & 18.6 \\
\hline & 15 & MSP & 161.40 & 6.16 & 2.37 & 19.48 & 19.48 & 19.27 \\
\hline \multirow{3}{*}{3} & 16 & EI & 177 & 5.4 & 3 & 0.004 & 19.39 & 18.26 \\
\hline & 17 & PI & 177 & 5.52 & 3 & 0.022 & 19.37 & 18.26 \\
\hline & 18 & MSP & 163.09 & 6.29 & 2.43 & 18.96 & 18.96 & 19.08 \\
\hline \multirow{3}{*}{4} & 19 & EI & 160 & 8 & 3 & $1.2 \times 10^{-4}$ & 18.24 & 18.34 \\
\hline & 19 & PI & 160 & 8 & 3 & 0.001 & 18.24 & 18.34 \\
\hline & 20 & MSP & 164.32 & 6.24 & 2.38 & 19.02 & 19.02 & 18.88 \\
\hline \multirow{3}{*}{5} & 21 & EI & 160 & 5 & -3 & $5.13 \times 10^{-4}$ & 18.24 & 17.54 \\
\hline & 21 & PI & 160 & 5 & -3 & 0.004 & 18.24 & 17.54 \\
\hline & 22 & MSP & 163.02 & 6.29 & 2.38 & 18.97 & 18.97 & 18.81 \\
\hline \multirow{3}{*}{6} & 23 & EI & 160 & 8 & -3 & $1.1 \times 10^{-6}$ & 18.24 & 17.83 \\
\hline & 23 & PI & 160 & 8 & -3 & $1.2 \times 10^{-5}$ & 18.24 & 17.83 \\
\hline & 24 & MSP & 163.73 & 6.32 & 2.46 & 18.97 & 18.96 & 18.76 \\
\hline
\end{tabular}

According to the EI and PI filling criterion, the new sample points coincided in the fourth to the sixth filling process, and they were all distributed on the boundary of the given range. Starting from the third edition, the relative error between the predicted volume flow rate and the volume flow rate obtained by CFD was between $1 \%$ and $2 \%$. From the perspective of the filling factors of the three filling criteria, the MSP filling criterion in this study reached the convergence condition earlier than EI and PI. Still, it did not meet the optimization termination condition of this study.

\subsubsection{Convergence Stage}

The fill factors corresponding to the EI and PI filling criterion were far smaller than the given threshold, and the entire optimization process met the termination condition. The maximum EI was very close to zero, while the maximum PI was already zero. The improvement of the subsequent RKM prediction ability by continuing to fill in new samples was negligible. From the entire parallel filling process perspective, the parallel filling criterion based on RKM had good convergence in specific engineering applications. 
On the basis of the RKM established after the seventh parallel filling, the optimal design parameters obtained by the genetic algorithm corresponded to the 27th group of samples in Table 7. Figure 13 shows the spatial distribution of the volume flow rate predicted by RKM under different optimal design parameters. There was obvious interaction among the three design variables, and the optimal design parameters solved by genetic algorithm corresponded to the optimal design parameters in RKM one by one.

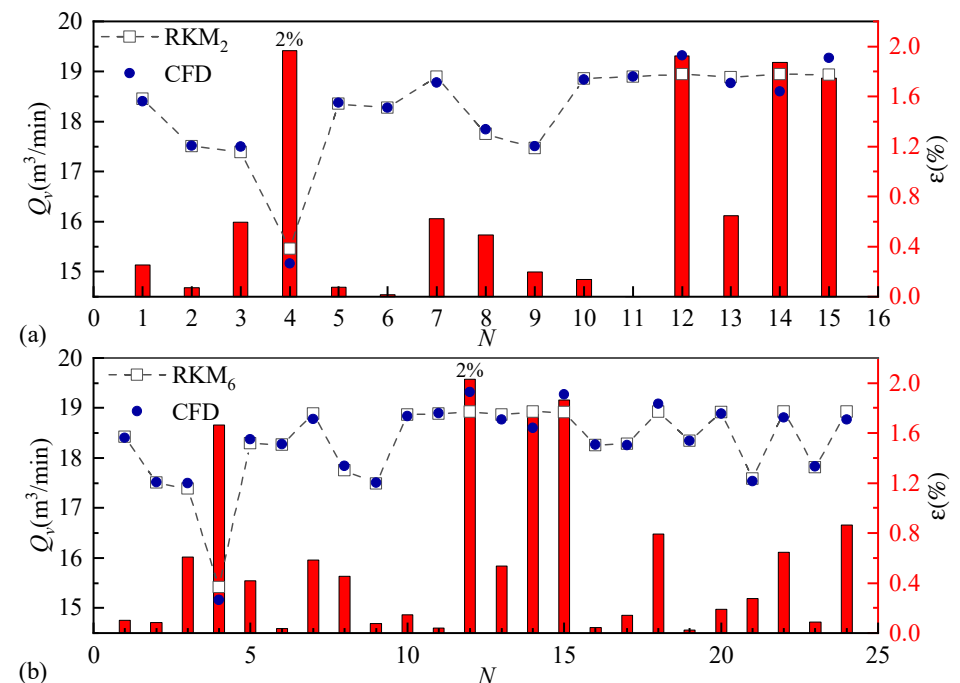

Figure 12. The prediction results of the sample points by RKM in the improvement stage: (a) the sample after the second filling; (b) the sample after the sixth filling.

Table 7. Filling process in the improvement stage.

\begin{tabular}{|c|c|c|c|c|c|c|c|c|}
\hline $\begin{array}{l}\text { Filling } \\
\text { Times }\end{array}$ & $\begin{array}{c}\text { Number of } \\
\text { Samples }\end{array}$ & $\begin{array}{l}\text { Filling } \\
\text { Criterion }\end{array}$ & $\begin{array}{l}\beta_{2} \\
\left(^{\circ}\right)\end{array}$ & $\underset{(\mathrm{mm})}{f}$ & $\begin{array}{l}\Delta \theta \\
\left({ }^{\circ}\right)\end{array}$ & Fill Factor & $\begin{array}{l}\text { Predictive } \\
\text { Value } \\
\left(\mathrm{m}^{3} / \mathrm{min}\right)\end{array}$ & $\begin{array}{c}\text { CFD } \\
\left(\mathrm{m}^{3} / \mathrm{min}\right)\end{array}$ \\
\hline \multirow{3}{*}{7} & 25 & EI & 177 & 8 & 3 & $8.9 \times 10^{-14}$ & 17.8 & 17.8 \\
\hline & 26 & PI & 177 & 5 & 0.18 & $2.5 \times 10^{-13}$ & 17 & 17.54 \\
\hline & 27 & MSP & 162.88 & 6.33 & 2.41 & 18.93 & 18.94 & 18.98 \\
\hline \multirow{3}{*}{8} & 28 & EI & 160 & 6.52 & -3 & $1.1 \times 10^{-13}$ & 18.05 & 18.13 \\
\hline & 28 & PI & 160 & 6.52 & -3 & $5.4 \times 10^{-12}$ & 18.05 & 18.13 \\
\hline & 29 & MSP & 162.28 & 6.23 & 2.43 & 18.94 & 18.94 & 18.97 \\
\hline \multirow{3}{*}{9} & 30 & EI & 177 & 5 & -3 & $4 \times 10^{-21}$ & 17.36 & 17.36 \\
\hline & 30 & PI & 177 & 5 & -3 & 0 & 17.36 & 17.36 \\
\hline & 31 & MSP & 163.46 & 6.23 & 2.46 & 18.94 & 18.94 & 18.97 \\
\hline
\end{tabular}

The statistical significance of the three design parameters was determined by variance analysis (ANOVA) to analyze further the influence of three design variables on volume flow rate. Figure 14 shows the Pareto chart of the ANOVA with an impact level of 0.05. The three design parameters of the blade had a robust nonlinear relationship with the $Q_{v}$. Since the influence of higher-order terms on $Q_{v}$ is generally tiny, only the first and second-order terms were given to reflect the immediate impact and interaction of design parameters. The second-order term of the maximum curvature $f$ had the most significant on $Q_{v}$, and it had a negative effect. For the first-order term, the blade exit angle and maximum curvature had a negative effect, and the blade exit position had a positive effect. The interaction between the design parameters occupied a more significant proportion. The interaction between the exit angle and the maximum curvature had an enormous influence and was a negative effect. For this reason, in the blade design process of the range hood, the interaction between the various design parameters should be comprehensively considered. 

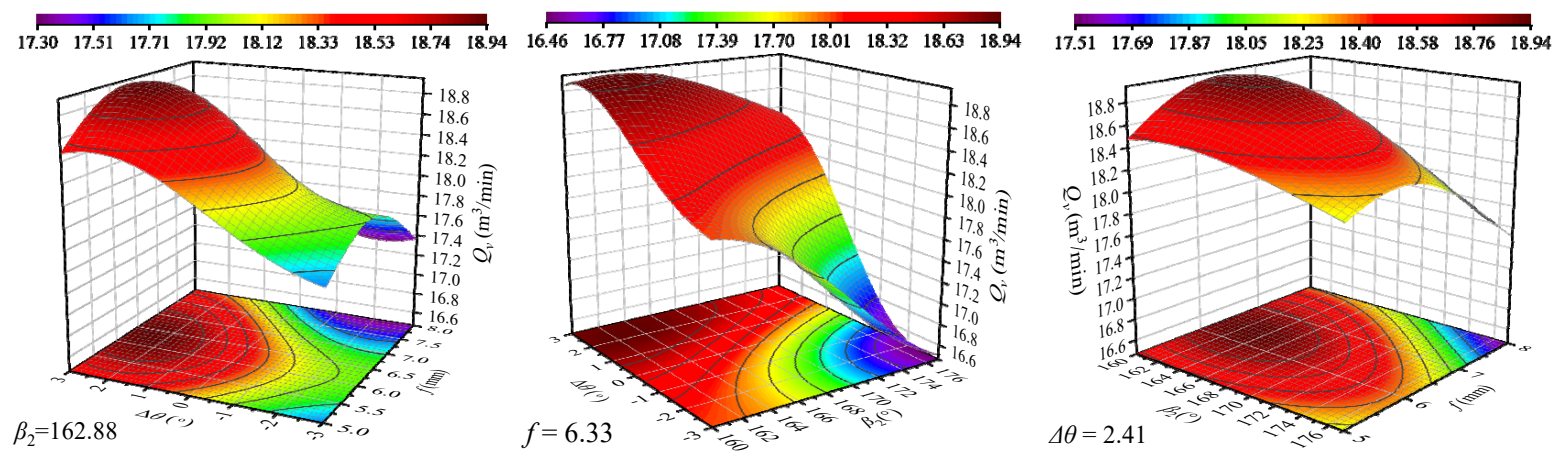

Figure 13. Spatial distribution of RKM under different optimal design parameters.

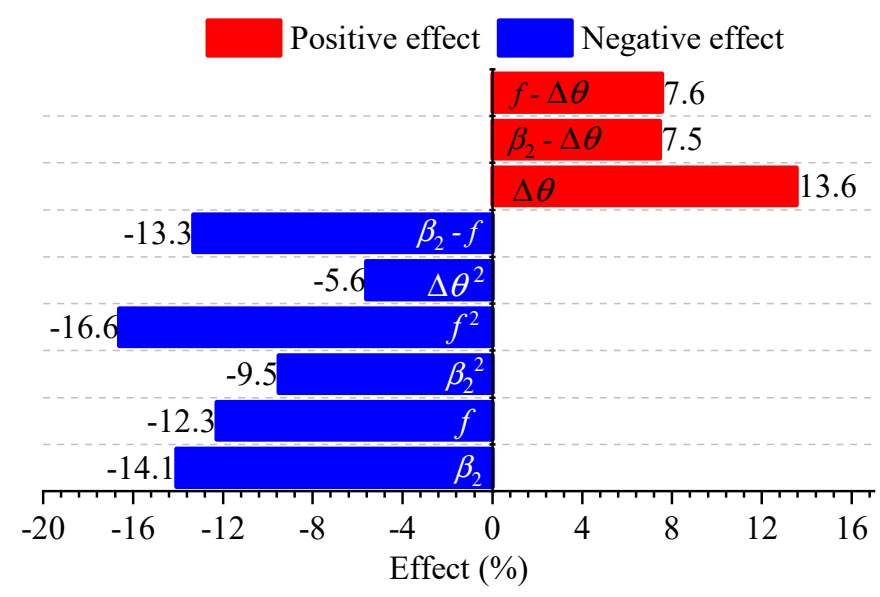

Figure 14. Pareto chart for ANOVA.

\subsection{Experimental Verification}

In the experimental verification stage, the input power of the AC motor was controlled by adjusting the voltage, and a stroboscope measured the speed of the impeller to ensure that the speed of the impeller under each working condition was consistent with the prototype. In the parallel filling process, the volume flow rate of the 12th sample in the initial stage was $0.34 \mathrm{~m}^{3} / \mathrm{min}$ higher than that of the 27th sample in the convergence stage. The two optimal impellers were both experimentally verified to determine the role of RKM in filtering CFD noise. Figure 15 shows the comparison of the performance curves of the optimal sample of RKM (the 27th sample in Table 7) and the CFD (the 27th sample in Table 5) with the prototype.

The blade profile and performance curve corresponding to the optimal sample of RKM and the optimal sample of CFD were very close. Compared with the prototype, the optimized blades improved the range hood's performance under large flow conditions. Still, the efficiency under small flow conditions was reduced to a certain extent. Under BVP conditions, the volume flow rate of the optimal sample of RKM and the optimal sample of CFD were increased by $2.9 \%$ and 3\%, respectively, and the efficiency increased by $1.8 \%$ and $2 \%$, respectively. Since the relative errors between the volume flow of the optimal sample of RKM and the optimal sample of CFD and the experimental results were $1.5 \%$ and $0.4 \%$, it fully verified the role of RKM in filtering CFD noise during the optimization of the range hood.

Since the relative error between the experiment and CFD of the optimal sample of RKM was only $0.4 \%$, the following internal flow state was analyzed by the CFD results of the optimal sample of RKM. The radial velocity cloud diagram of the impeller outlet was used to analyze the degree of improvement of the airflow at the impeller outlet to find why the optimized blades increase the volume flow rate of the range hood. 


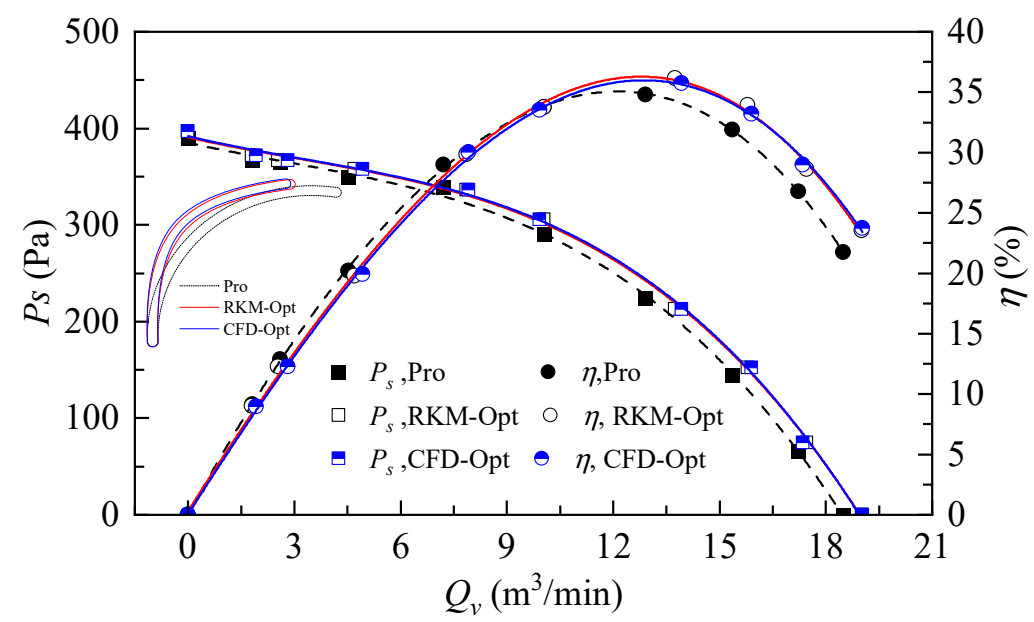

Figure 15. Comparison of the performance curves of the optimal sample of RKM and the CFD with the prototype.

\subsection{Flow Field Analysis}

Figure 16 shows a plane cloud diagram of the radial velocity of the impeller exit ring section expanded in the circumferential direction. The radial velocity of the impeller outlet was very unevenly distributed along the axial direction ( $z$-direction) and the circumferential direction ( $\theta$ direction) of the impeller. The larger radial velocity distribution area was mainly concentrated in the area near the circumferential direction $\theta$ from $130^{\circ}$ to $360^{\circ}$, and the axial $\mathrm{z}$ was $85 \mathrm{~mm}$ (the middle of the impeller), indicating that this area was the main flow area of the impeller. The radial velocity of the impeller corresponding to the area near the volute tongue $\left(\theta=120^{\circ}\right)$ was negative, indicating that the airflow in this area flowed into the impeller in the opposite direction. From the comparison of the radial velocity before and after optimization, the radial velocity in the mainstream area increased significantly after optimization, and the distribution range was wider than the prototype.

The middle section of the impeller $(z=85 \mathrm{~mm})$ was used to analyze the flow inside the impeller in detail. From the distribution of the radial velocity $V_{r}$ in Figure 17a along the circumferential angle, the optimized impeller, in addition to the increase in the return flow corresponding to the volute tongue, the radial velocity of the corresponding impeller region downstream of the volute tongue, was smaller than the original impeller. However, in the mainstream area, the increase in the radial velocity of the impeller outlet was more obvious than the negative effect brought by the backflow and the downstream region of the volute tongue, and the radial velocity and volume flow rate of the outlet section of the optimized impeller increased.

The exterior of Figure 17a shows the velocity distribution and streamline diagram. It can be found that in each flow channel of the impeller, vortices of a certain scale were distributed and attached to the suction surface of the blade. Figure 17b shows the flow details of the blade passage in the main flow area near $\theta=300^{\circ}$. The inlet airflow of the impeller flowed into the impeller along the positive angle of attack $i$. Still, the inlet velocity of the optimized impeller was significantly higher than that of the original impeller.

Figure 18 shows the change of the impeller cross-sectional area along the impeller diameter before and after optimization. The evolution of the flow area of the impeller after optimization was similar to the trend of the original impeller, and the flow area was first increased and then decreased. When the airflow flowed into the impeller at a large angle of attack, the flow area of the impeller increased, and the distribution range was larger after optimization, with there being enough flow area to make the airflow flow along the pressure surface of the blade. Therefore, the vortex was more easily adsorbed on the suction surface of the leading edge, and the further development of the vortex was inhibited. When the airflow was ready to go out of the impeller, the optimized impeller 
rapidly reduced the flow cross-section area, further increasing the speed of the airflow at the exit of the impeller.

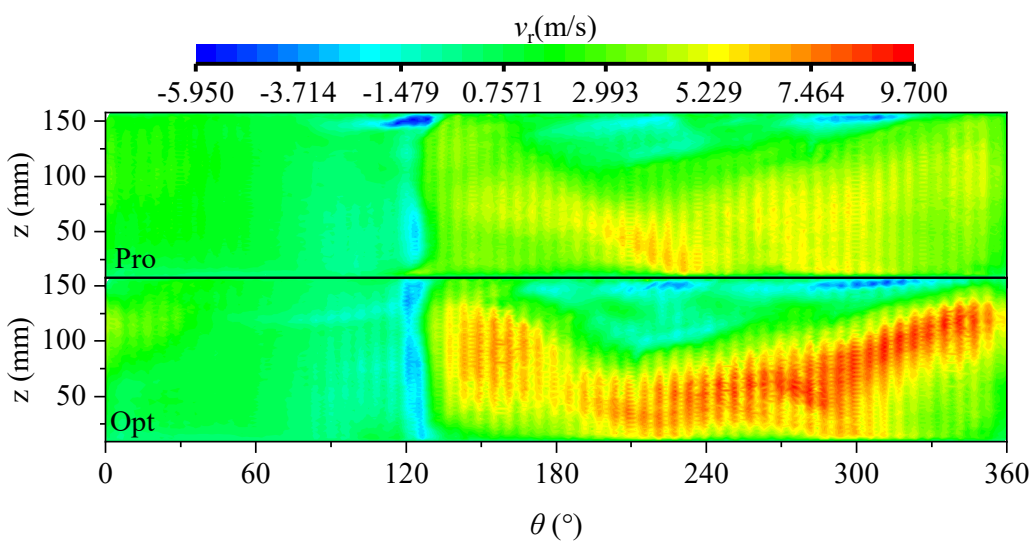

Figure 16. The contrast of radial velocity at the impeller outlet.

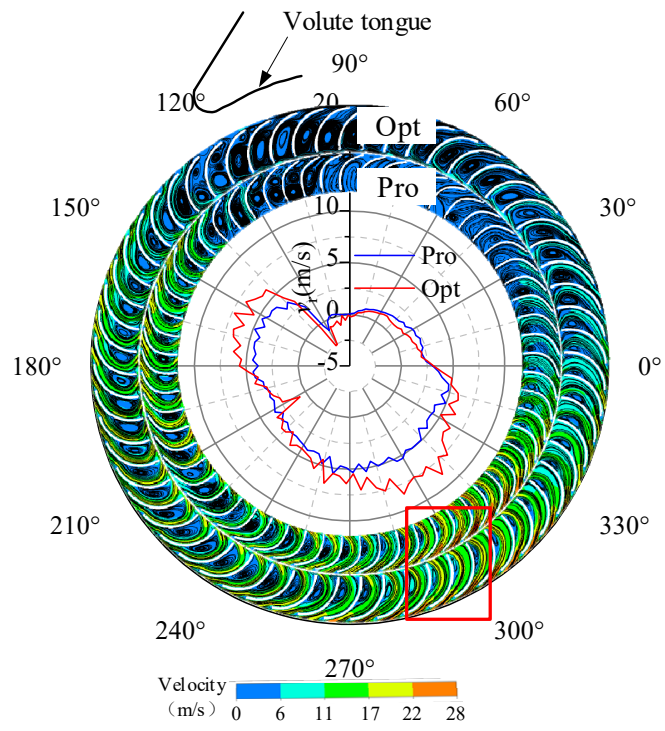

(a)

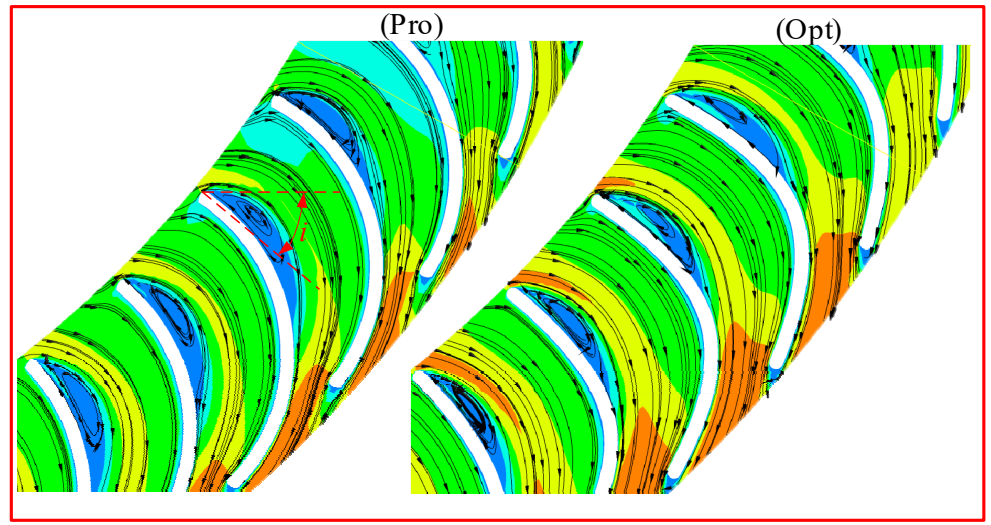

(b)

Figure 17. Comparison of velocity distribution in the middle section: (a) the radial velocity at the exit of the impeller inside and the velocity distribution and streamline diagram outside; (b) the partial enlargement of the velocity distribution and streamline diagram.

The airflow in the area near the volute tongue is shown in Figure 19. The airflow near the volute tongue can be divided into three parts: the airflow discharged from the volute $Q_{\text {dis, }}$, the airflow recirculated into the volute $Q_{\text {rec }}$, and the airflow reversed into the impeller $Q_{\text {rev2 }}$. At the inlet of the impeller, there was also a reverse flow of air flowing out of the impeller $Q_{\text {rev1. }}$. The airflow discharged from the volute was the effective volume flow rate, which corresponded to the volume flow rate of the range hood. The circulating flow and reverse flow were related to the loss of shaft power, corresponding to the useless volume flow rate.

The circulating volume flow rate ratio and the reverse volume flow to the effective volume flow rate are the circulating flow coefficient and the reverse flow coefficient [33]. As shown in Figure 20, the optimized circulating volume flow rate increased slightly, but the effective volume flow rate increased significantly, resulting in a slight decrease in the circulating flow coefficient. The reverse volume flow rate at the outlet of the optimized impeller was increased dramatically, which indicated that the matching relationship between 
the optimized impeller and the volute tongue became worse than the prototype. However, the optimized impeller significantly reduced the reverse flow at the impeller inlet, which substantially improved the air intake environment of the impeller.

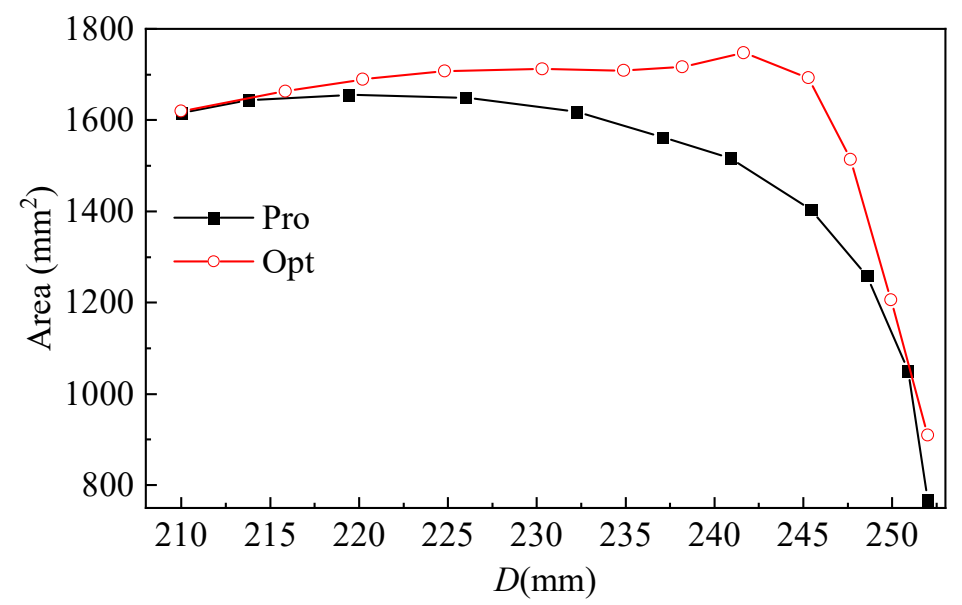

Figure 18. Change of cross-sectional area with diameter $D$.

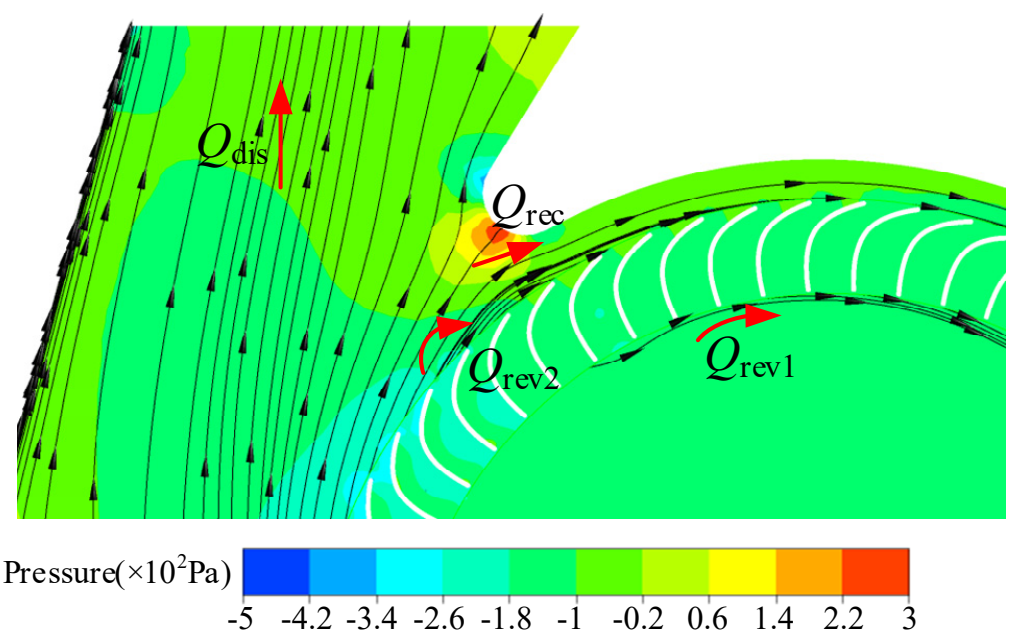

Figure 19. Airflow in the area near the volute tongue.

The static pressure recovery coefficient $C_{p}$ and the total pressure loss coefficient $K_{p}$ [34] can be used to measure the matching relationship between the impeller and the volute. The higher the $C_{p}$, the greater the ability of the volute to convert the kinetic energy of the high-speed airflow into static pressure more substantially. The lower the $K_{p}$, the smaller the flow loss of the airflow inside the volute. The $K_{p}$ and $C_{p}$ of the volute are defined as

$$
\left\{\begin{aligned}
K_{p} & =\frac{P_{i m p, t}-P_{v o l, t}}{P_{i m p},-P_{v o l, s}} \times 100 \% \\
C_{p} & =\frac{P_{v o l, s}-P_{i m p, s}}{P_{i m p, t}-P_{v o l, s}} \times 100 \%
\end{aligned}\right.
$$

where $P_{i m p, t}$ and $P_{i m p, s}$ represent the total pressure and static pressure at the impeller outlet, respectively. $P_{v o l, t}$ and $P_{v o l, s}$ represent the total pressure and static pressure at the volute outlet, respectively.

As shown in Figure 21, the optimized static pressure recovery coefficient $C_{p}$ increased from 41.88 to 43.83 , indicating that the optimized impeller makes the volute have better pressure diffusion performance. The optimized total pressure loss coefficient reduced from 15.35 to 8.5 , which shows that the optimized impeller can significantly reduce the flow loss 
inside the volute. Therefore, the matching relationship between the optimized impeller and the existing volute was improved.

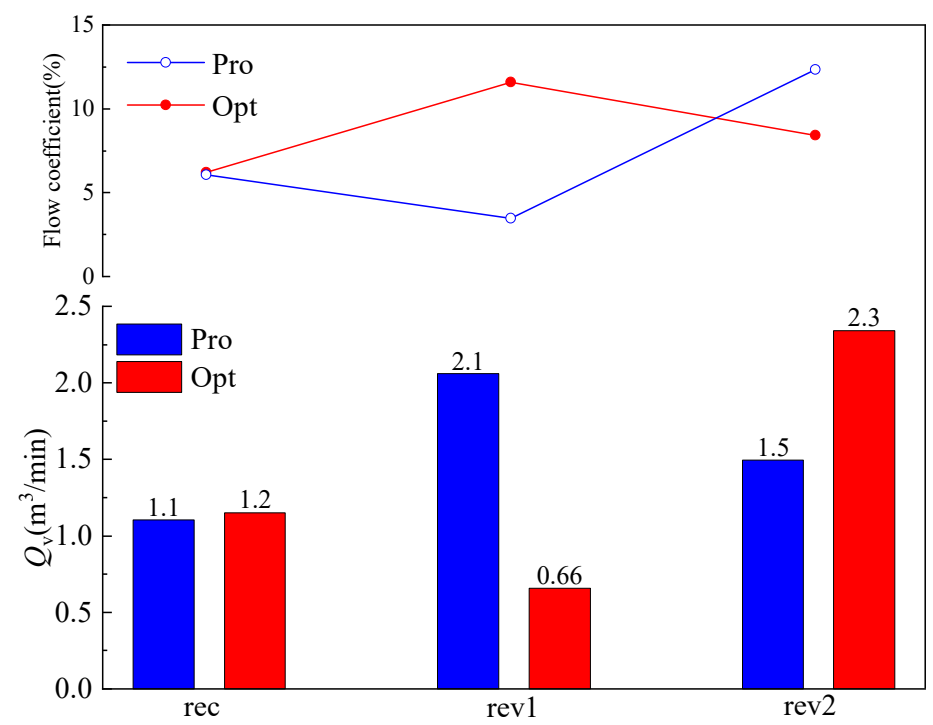

Figure 20. Airflow in the area near the volute tongue.

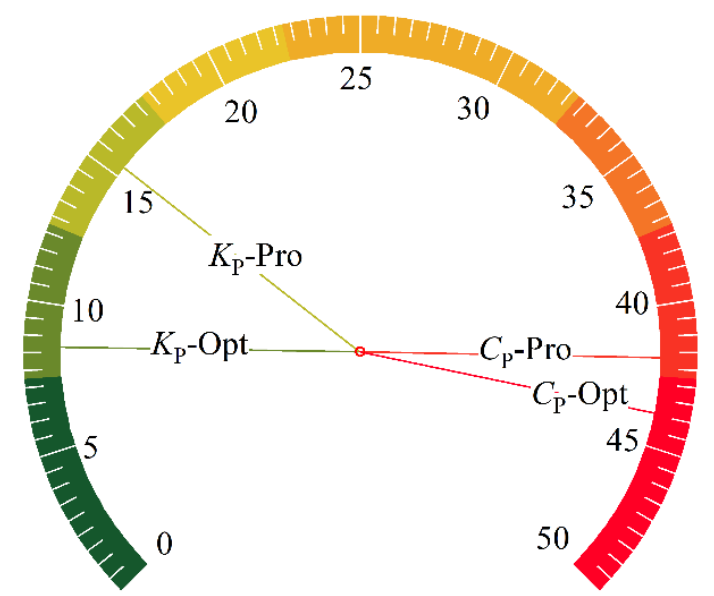

Figure 21. Comparison of the $K_{p}$ and $C_{p}$ before and after optimization.

\section{Flow Field Prediction}

The optimization part verifies that RKM can accurately predict the volume flow rate $Q_{v}$, but whether RKM can accurately predict the flow field is still unknown. In the case of the constant cross-sectional area of the volute exit, the volume flow rate of the range hood depends on the normal velocity of the exit cross-section of the volute. The volume flow rate $Q_{v}$ in the numerical simulation depends on the grid area $\vec{A}$ of the exit section of the volute and the normal velocity $\vec{v}$ :

$$
Q_{v}=\int \vec{v} \cdot d \vec{A}=\sum_{i=1}^{n} \vec{v}_{i} \cdot \vec{A}
$$

where $n$ represents the number of grids in the exit section of the volute. In this study, since only the grid of the impeller was changed and the grid of the volute region was constant, the volume flow rate depended on the normal velocity of each grid.

The prediction process is shown in Figure 22. The $n, m$, and $v_{i j}$ represent the number of the grid, the number of training samples, and the normal velocity of the ith grid in group $j$, respectively. The first six parallel filling samples were used as training samples 
(a total of 24 sets of samples, $m=24$ ), and the optimal sample obtained in the seventh parallel filling process (the 27 th set of samples) was used as the prediction sample. RKM was used to predict the normal velocity of the volute outlet section of the optimal sample. The normal velocity of the volute outlet section of the previous 24 groups of samples and the corresponding three design parameters were used as training data. Since there were 1143 grids $(n=1143)$ in the exit section of the volute, 1143 RKMs needed to be trained. When the training was completed, the three design parameters of the optimal sample were used as input to predict the normal velocity of each grid cell of the volute exit section of the optimal sample.

Data

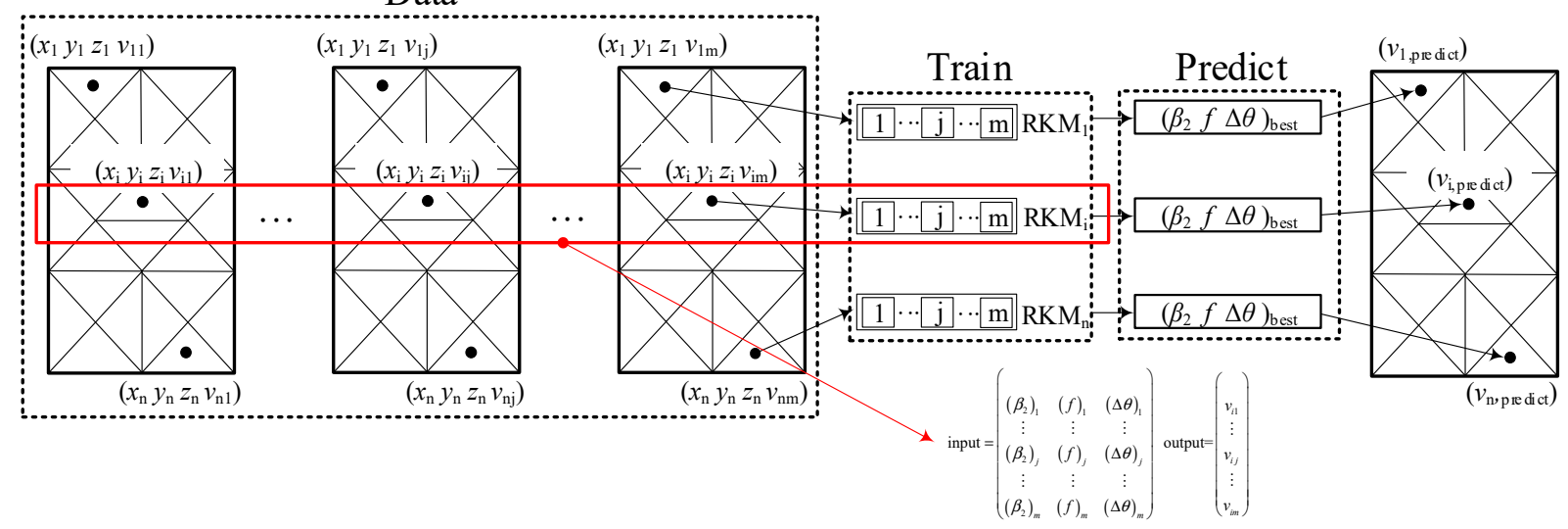

Figure 22. Flow field prediction process.

The predicted result is shown in Figure 23. As shown in Figure 23a, the normal velocity changed with the grid cell. RKM could not accurately predict the normal velocity of each grid at the exit of the volute. However, the predicted value of the normal speed of each grid and the calculated value of CFD were fitted with a cubic polynomial to obtain the changing trend of the normal speed. The predicted trend of RKM was highly consistent with the trend of CFD calculation. Figure 23b shows the comparison of the normal velocity contour. The normal velocity of the CFD was very close to the predicted normal velocity cloud image. The volumetric flow rate corresponding to the statistically predicted flow field was 18.87 , and the relative error with CFD was only $0.5 \%$. Therefore, RKM can accurately predict the volume flow because it can accurately predict the changing trend of the normal velocity of the exit section.

The impeller exit section with more drastic changes in radial velocity was used for prediction to verify further whether RKM can accurately predict the trend of physical quantities in other departments. The interface between the impeller outlet and the volute whose mesh had not changed was selected. There were 10,530 grids $(n=10,530)$, and therefore 10,530 RKMs needed to be trained. Figure 24 shows the prediction results of the radial velocity of the impeller exit section. The trend predicted by RKM was highly consistent with the trend calculated by CFD. From the comparison of the radial velocity contour in Figure 24b, in the volute tongue area A, the distribution of the front disc area B of the impeller and the mainstream area $C$ were very close. Still, the distribution of details was very different. Judging from the prediction results of the volute and impeller section, we found that RKM can accurately predict the changing trend of the relevant physical quantities of the range hood. 


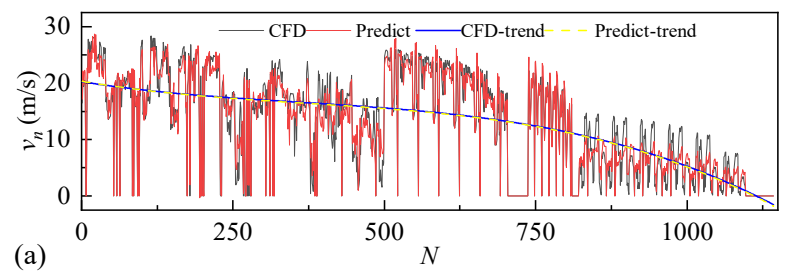

(b)

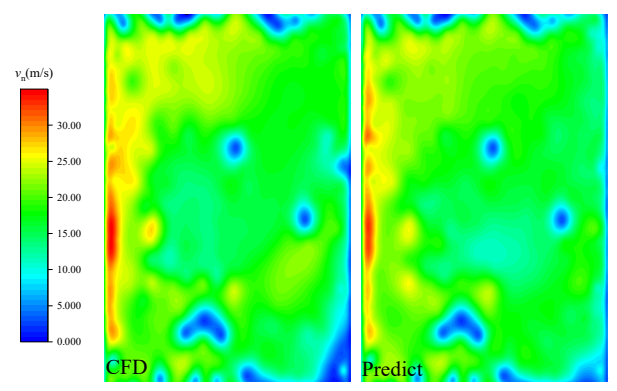

Figure 23. The prediction results of the normal velocity of the exit section of the volute: (a) the change of the normal velocity with the grid; (b) the comparison of the normal velocity contour.

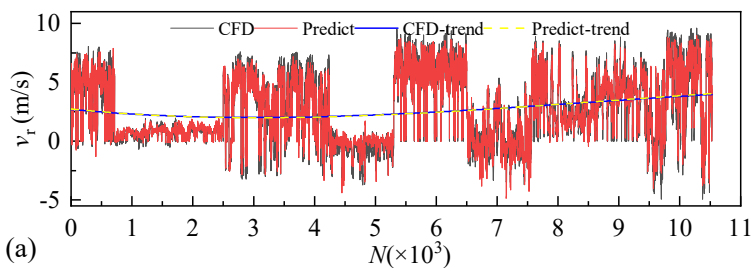

(a)

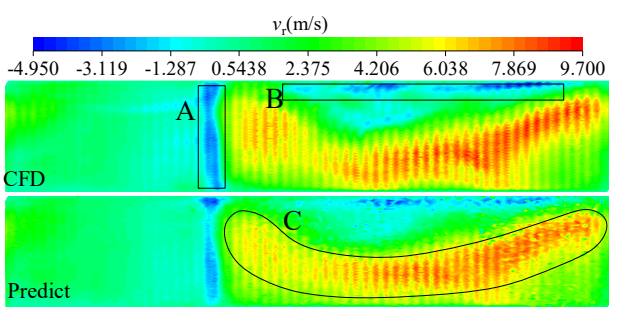

Figure 24. The prediction results of the radial velocity of the impeller outlet section: (a) the radial velocity changes with the grid; (b) the comparison of the radial velocity contour.

\section{Conclusions}

The parallel filling criterion of EI, PI, and MSP of RKM in this study had good convergence in the optimization process of the volume flow rate. According to the changes of $\mathrm{EI}_{\max }, \mathrm{PI}_{\max }$, and $\triangle \mathrm{MSP}$ and the predictive ability of RKM in the parallel filling process, the entire filling process can be divided into an initial stage, an improvement stage, and a convergence stage. There was a strong nonlinear relationship between the three design parameters (blade exit angle $\beta_{2}$, variation of blade outlet position $\Delta \theta$, and the maximum curvature $f$ of the blade camber line) and the volume flow rate $Q_{v}$. The second-order terms of the maximum curvature $\mathrm{f}$ had the greatest impact on $Q_{v}$ through the analysis of variance, and it had a negative effect. The interaction among the three design parameters accounted for a large proportion. In the process of blade design, the interaction between each design parameter should be considered comprehensively.

Through the experimental verification, we found that the blade profile and performance curve of the optimal sample of RKM and CFD were very close. However, the relative error between the volume flow rate of the optimal sample of RKM and the experiment was only $0.4 \%$, which fully verified the role of RKM in filtering CFD noise during the optimization. Under BVP conditions, the volume flow rate and efficiency of the RKM optimal sample were increased by $2.9 \%$ and $2 \%$, respectively. 
The optimal sample of RKM made the radial velocity of the impeller outlet significantly higher than that of the prototype. The improvement effect of the flow state in the mainstream area of the impeller and the inlet conditions of the impeller was more obvious than the negative effect brought by the return flow and the downstream region of the volute tongue. After the optimization, the change characteristics of the impeller cross-sectional area made it easier for the vortex in the blade channel to be adsorbed on the suction surface of the blade leading edge. The further development of the vortex was inhibited. The matching relationship between the optimized impeller and the volute was also improved, making the volute have better pressure diffusion performance than the prototype and leading to reduction in internal flow loss. Through the flow field prediction, the RKM can accurately predict the range hood's performance because it accurately predicts the changing trend more than it accurately predicts the physical quantity on each grid of the CFD.

Author Contributions: Conceptualization, Q.X. and J.W.; methodology, Q.X.; software, Q.X. and Y.D.; validation, X.S., L.W. and J.W.; formal analysis, Q.X.; investigation, Q.X.; resources, Y.D.; data curation, B.J.; writing—original draft preparation, Q.X.; writing—review and editing, Q.X.; visualization, Q.X.; supervision, J.W.; project administration, B.J. and X.S.; funding acquisition, J.W. All authors have read and agreed to the published version of the manuscript.

Funding: This research was supported by the Fundamental Research Funds for the Central Universities, HUST: 2021JYCXJJ048.

Institutional Review Board Statement: Not applicable.

Informed Consent Statement: Not applicable.

Data Availability Statement: Not applicable.

Acknowledgments: The authors thank the SCTS/CGCL HPCC of HUST for providing computing resources and technical support. The authors also appreciate all other scholars for their advice and assistance in improving this article.

Conflicts of Interest: The authors declare no conflict of interest.

\section{References}

1. Akbari, G.; Montazerin, N.; Akbarizadeh, M. Stereoscopic particle image velocimetry of the flow field in the rotor exit region of a forward-blade centrifugal turbomachine. Proc. Inst. Mech. Eng. Part A J. Power Energy 2012, 226, 163-181. [CrossRef]

2. Liu, H.; Jiang, B.; Wang, J.; Yang, X.; Xiao, Q. Numerical and experimental investigations on non-axisymmetric D-type inlet nozzle for a squirrel-cage fan. Eng. Appl. Comput. Fluid Mech. 2021, 15, 363-376. [CrossRef]

3. Keane, A.; Forrester, A.; Sóbester, A. Engineering Design via Surrogate Modelling: A Practical Guide; John Wiley \& Sons: Chichester, UK, 2008. [CrossRef]

4. Gao, X.; Zhu, H.; Zhang, H.; Sun, B.; Qin, Z.; Tian, Y. CFD Optimization Process of a Lateral Inlet/Outlet Diffusion Part of a Pumped Hydroelectric Storage Based on Optimal Surrogate Models. Processes 2019, 7, 204. [CrossRef]

5. Elfarra, M.A. Optimization of helicopter rotor blade performance by spline-based taper distribution using neural networks based on CFD solutions. Eng. Appl. Comput. Fluid Mech. 2019, 13, 833-848. [CrossRef]

6. Ma, S.-B.; Kim, S.; Kim, J.-H. Optimization Design of a Two-Vane Pump for Wastewater Treatment Using Machine-Learning-Based Surrogate Modeling. Processes 2020, 8, 1170. [CrossRef]

7. De Paepe, W.; Pappa, A.; Coppitters, D.; Carrero, M.M.; Tsirikoglou, P.; Contino, F. Recuperator Performance Assessment in Humidified Micro Gas Turbine Applications Using Experimental Data Extended with Preliminary Support Vector Regression Model Analysis. J. Eng. Gas. Turbines Power 2021, 143, 071030. [CrossRef]

8. Zhang, W.; Li, L.; Zhang, B.; Xu, X.; Zhai, J.; Wang, J. A Closed-Loop Optimized System with CFD Data for Liquid Maldistribution Model. Processes 2020, 8, 1332. [CrossRef]

9. Forrester, A.I.J.; Keane, A.J.; Bressloff, N.W. Design and Analysis of "Noisy" Computer Experiments. AIAA J. 2006, 44, 2331-2339. [CrossRef]

10. Zhang, Y.; Han, Z.-H.; Zhang, K.-S. Variable-fidelity expected improvement method for efficient global optimization of expensive functions. Struct. Multidiscip. Optim. 2018, 58, 1431-1451. [CrossRef]

11. Ginsbourger, D.; Le Riche, R.; Carraro, L. A multi-points criterion for deterministic parallel global optimization based on kriging. In Proceedings of the International Conference on Nonconvex Programming, Local and Global Approaches, Rouen, France, 1-30 March 2008. 
12. Booker, A.J.; Dennis, J.E.; Frank, P.D.; Torczon, V.; Trosset, M.W. A Rigorous Framework for Optimization of Expensive Functions by Surrogates. Struct. Optim. 1998, 17, 1-13. [CrossRef]

13. Jones, D.R.; Schonlau, M.; Welch, W.J. Efficient Global Optimization of Expensive Black-Box Functions. J. Glob. Optim. 1998, 13, 455-492. [CrossRef]

14. Jeong, S.; Murayama, M.; Yamamoto, K. Efficient Optimization Design Method Using Kriging Model. J. Aircr. 2005, 42, 413-420. [CrossRef]

15. Jones, D.R. A Taxonomy of Global Optimization Methods Based on Response Surfaces. J. Glob. Optim. 2001, 21, 345-383. [CrossRef]

16. Sasena, M.J.; Papalambros, P.; Goovaerts, P. Exploration of Metamodeling Sampling Criteria for Constrained Global Optimization. Eng. Optim. 2002, 34, 263-278. [CrossRef]

17. Laurenceau, J.; Meaux, M.; Montagnac, M.; Sagaut, P. Comparison of Gradient-Based and Gradient-Enhanced Response-SurfaceBased Optimizers. AIAA J. 2010, 48, 981-994. [CrossRef]

18. Liu, J.; Han, Z.; Song, W. Comparison of infill sampling criteria in kriging-based aerodynamic optimization. In Proceedings of the 28th Congress of the International Council of the Aeronautical Sciences, Brisbane, Australia, 23-28 September 2012.

19. Hengl, T.; Heuvelink, G.B.; Rossiter, D.G. About regression-kriging: From equations to case studies. Comput. Geosci. 2007, 33, 1301-1315. [CrossRef]

20. Bonakdari, H.; Baghalian, S.; Nazari, F.; Fazli, M. Numerical Analysis and Prediction of the Velocity Field in Curved Open Channel Using Artificial Neural Network and Genetic Algorithm. Eng. Appl. Comput. Fluid Mech. 2011, 5, 384-396. [CrossRef]

21. Raissi, M.; Yazdani, A.; Karniadakis, G.E. Hidden fluid mechanics: Learning velocity and pressure fields from flow visualizations. Science 2020, 367, 1026-1030. [CrossRef] [PubMed]

22. Rabault, J.; Kuchta, M.; Jensen, A.; Réglade, U.; Cerardi, N. Artificial Neural Networks trained through Deep Reinforcement Learning discover control criterion for active flow control. J. Fluid Mech. 2019, 865, 281-302. [CrossRef]

23. Li, B.; Yang, Z.; Zhang, X.; He, G.; Deng, B.-Q.; Shen, L. Using machine learning to detect the turbulent region in flow past a circular cylinder. J. Fluid Mech. 2020, 905, 1-26. [CrossRef]

24. Yang, X.; Jiang, B.; Wang, J.; Huang, Y.; Yang, W.; Yuan, K.; Shi, X. Multi-objective optimization of dual-arc blades in a squirrel-cage fan using modified non-dominated sorting genetic algorithm. Proc. Inst. Mech. Eng. Part A J. Power Energy 2020, 234, 1053-1068. [CrossRef]

25. Wang, K.; Ju, Y.; Zhang, C. Experimental and numerical investigations on effect of blade trimming on aerodynamic performance of squirrel cage fan. Int. J. Mech. Sci. 2020, 177, 105579. [CrossRef]

26. Yanyan, D.; Wang, J.; Wang, W.; Jiang, B.; Xiao, Q.; Ye, T. Multi-condition optimization of a cross-flow fan based on the maximum entropy method. Proc. Inst. Mech. Eng. Part A J. Power Energy 2021. [CrossRef]

27. Kim, J.-H.; Choi, J.-H.; Husain, A.; Kim, K.-Y. Performance enhancement of axial fan blade through multi-objective optimization techniques. J. Mech. Sci. Technol. 2010, 24, 2059-2066. [CrossRef]

28. Menter, F.R. Two-equation eddy-viscosity turbulence models for engineering applications. AIAA J. 1994, 32, 1598-1605. [CrossRef]

29. Giunta, A.; Wojtkiewicz, S.; Eldred, M. Overview of Modern Design of Experiments Methods for Computational Simulations (Invited). In Proceedings of the 41st Aerospace Sciences Meeting and Exhibit, Reno, NV, USA, 6-9 January 2003. [CrossRef]

30. Morris, M.D.; Mitchell, T.J. Exploratory designs for computational experiments. J. Stat. Plan. Inference 1995, 43, 381-402. [CrossRef]

31. Forrester, A.I.; Keane, A. Recent advances in surrogate-based optimization. Prog. Aerosp. Sci. 2009, 45, 50-79. [CrossRef]

32. Leary, S.; Keane, A. A parallel updating scheme for approximating and optimizing high fidelity computer simulations. Struct. Multidiscip. Optim. 2004, 27, 371-383. [CrossRef]

33. Wang, K.; Ju, Y.; Zhang, C. A Quantitative Evaluation Method for Impeller-Volute Tongue Interaction and Application to Squirrel Cage Fan with Bionic Volute Tongue. J. Fluids Eng. 2019, 141, 081104. [CrossRef]

34. Wen, X.; Mao, Y.; Yang, X.; Qi, D. Design Method for the Volute Profile of a Squirrel Cage Fan with Space Limitation. J. Turbomach. 2016, 138, 081001. [CrossRef] 